\title{
A Universal Scheme for Wyner-Ziv Coding of Discrete Sources
}

\author{
Shirin Jalali, Student Member, IEEE, Sergio Verdú, Fellow, IEEE, and Tsachy Weissman, Senior Member, IEEE
}

\begin{abstract}
We consider the Wyner-Ziv (WZ) problem of lossy compression where the decompressor observes a noisy version of the source, whose statistics are unknown. A new family of WZ coding algorithms is proposed and their universal optimality is proven. Compression consists of sliding-window processing followed by Lempel-Ziv (LZ) compression, while the decompressor is based on a modification of the discrete universal denoiser (DUDE) algorithm to take advantage of side information. The new algorithms not only universally attain the fundamental limits, but also suggest a paradigm for practical $\mathrm{WZ}$ coding. The effectiveness of our approach is illustrated with experiments on binary images, and English text using a low complexity algorithm motivated by our class of universally optimal WZ codes.
\end{abstract}

Index Terms-Discrete denoising, rate-distortion function, sliding-window coding, universal algorithm, Wyner-Ziv coding.

\section{INTRODUCTION}

C ONSIDER the basic setup shown in Fig. 1 consisting of a source with unknown statistics driving a known discrete memoryless channel (DMC), and a decoder that receives a compressed version of the source in addition to the noisy channel output. The goal is to minimize the distortion between the source and the reconstructed signal by optimally designing the encoder and decoder. This is the problem of rate-distortion coding with decoder side information, commonly known as Wyner-Ziv compression after the seminal paper [1]. Even without side information, the problem of finding universal practical schemes that get arbitrarily close to a given point on the rate-distortion curve is notoriously challenging (see [2]-[4] for recently proposed practical schemes). Even when the discrete source distribution is known, no practical scheme is currently known to approach the rate-distortion function when the source has memory. Other than the region of low distortion, the rate-distortion function is not known even for a binary Markov source (see [5]-[7]).

As an example of practical motivation for the setup shown in Fig. 1, consider the problem of audio/video broadcasting where

Manuscript received February 28, 2008; revised November 19, 2009. Current version published March 17, 2010. The work of S. Jalali was supported by Stanford Graduate Fellowship. The work of S. Verdú was supported by the NSF under Grant CCR-0312839, National Science Foundation, Theoretical Foundations Research Grants CCF-0635154 and CCF-0728445. The work of T. Weissman was supported by an NSF CAREER grant.

S. Jalali is with the Center for the Mathematics of Information, California Institute of Technology, Pasadena, CA 91125 USA (e-mail: shirin@ caltech.edu).

$\mathrm{S}$. Verdú is with the Department of Electrical Engineering, Princeton University, Princeton, NJ 08544 USA (e-mail: verdu @ princeton.edu).

T. Weissman is with the Department of Electrical Engineering, Stanford University, Stanford, CA 94305 USA (e-mail: tsachy@ stanford.edu).

Communicated by H. Yamamoto, Associate Editor for Shannon Theory.

Color version of Figure 1 in this paper is available online at http://ieeexplore. ieee.org.

Digital Object Identifier 10.1109/TIT.2010.2040889

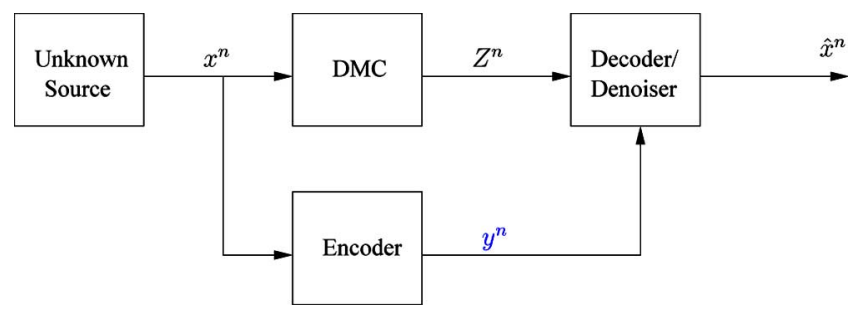

Fig. 1. The basic setup of WZ coding.

in addition to the analog signal, the decoder has access to some additional information transmitted in a digital channel for instance. In such setup, a legacy receiver only observes the output of the channel, while a more sophisticated receiver in addition has access to coded information which helps boosting reproduction fidelity. Thus, we can view the setup as one of universal systematic channel coding where the added "redundancy" is received error-free.

An alternative view of this problem is as a denoising problem where the denoiser, in addition to the noise-corrupted data, has access to a fidelity-boosting (FB) sequence conveyed to it via a channel of capacity $R$. Both viewpoints are equivalent because the source/channel separation theorem [8] guarantees that there is no loss in separating the source coding and channel coding operations at least under certain sufficient conditions on source and channel [9]. Therefore, the encoder is able to send any information with entropy less than the channel capacity almost losslessly to the decoder. Consequently, although in practice we would often have a channel of capacity $R$, we simply consider the encoder-channel-decoder chain as a noiseless bit pipe of rate $R$.

Note that in these two viewpoints the role of the main signal and fidelity-boosting signal is interchanged. In this paper, we adopt the latter, and suggest a new algorithm for WZ coding of a source with unknown statistics. We show that, for stationary ergodic sources, the algorithm is asymptotically optimal in the sense that its average expected loss per symbol converges to the minimum attainable expected loss.

The encoder of the proposed algorithm consists of a slidingblock (SB) coder followed by Lempel-Ziv (LZ) compression [10]. SB lossy compression is shown in [11] to be able to perform as well as conventional lossy block compression. We extend this result to the WZ coding setup, and show that the same result holds in this case as well. The reason we use SB codes instead of block codes in our algorithm is the special type of decoder we employ. The decoder is based on a modification of the discrete universal denoiser (DUDE) algorithm [12], DUDE with FB (fbDUDE), to take advantage of the FB sequence. We prove 
that the optimality results of the original DUDE carry over to fbDUDE as well.

As mentioned before, in our setting we always assume that the channel transition matrix is known both to the encoder and the decoder. As argued in [12], the assumption of a known channel and an unknown signal is realistic in many practical scenarios. Furthermore, unlike the DUDE setting [12], in the setup of this paper the decoder can easily learn the channel, e.g., by having the encoder dedicate a negligibly small portion of its rate to describing the first few components of the source sequence which then act as a training sequence. Further still, if a modicum of feedback from decoder to encoder is allowed, then the encoder too can be informed of the channel arbitrarily precisely. Therefore, unlike in the DUDE setting where knowledge of the channel plays a key role [13], [14], in our setting, at least decoder knowledge of the channel is not crucial, and our schemes can be easily modified to accommodate channel uncertainty.

Some progress towards practical WZ coding schemes has been made in recent years, as seen, e.g., in [15]-[20]. The proposed schemes, however, operate under specific assumptions of a known (usually memoryless) source and side information channel. Practical schemes for more general source and/or channel characteristics have yet to be developed and, a fortiori, no practical universal schemes for this problem are known.

The problem of $\mathrm{WZ}$ coding of a source with unknown statistics was recently considered in [21], where existence of universal schemes in a setting similar to ours is established. In contrast, our schemes suggest a paradigm for WZ coding of discrete sources which is not only practical but is justified through universal optimality results.

The organization of the remainder of this paper is as follows. In Section II, the notation used throughout the paper is introduced. Section III presents fbDUDE, the extension of the DUDE denoising algorithm [12] to take advantage of a FB sequence, and shows how the asymptotic optimality of the original DUDE carries over to this case as well. Section IV proposes SB WZ codes and proves a result on their relationship to WZ block codes. In Section V, our new WZ coding algorithm is presented and its optimality is established. In Section VI we present some experimental results, concluding in Section VII with a brief discussion of possible extensions of this work. Outlines of the proofs are given in the main body, with the full proofs relegated to the Appendix .

\section{NOTATION}

Let $\mathcal{X}, \hat{\mathcal{X}}$, and $\mathcal{Z}$ denote the source, reconstructed signal, and channel output alphabets, respectively. In this paper, for simplicity, we restrict attention to

$$
\mathcal{X}=\hat{\mathcal{X}}=\mathcal{Z}=\left\{\alpha_{1}, \ldots, \alpha_{N}\right\}
$$

though our derivations and results carry over directly to nonidentical finite sets $\mathcal{X}, \hat{\mathcal{X}}$, and $\mathcal{Z}$. Bold low case symbols, e.g., $\mathbf{x}, \mathbf{y}, \mathbf{z}$, denote individual sequences. The discrete memoryless channel is described by its transition matrix $\Pi$, where $\Pi(i, j)$ denotes the probability of getting $\alpha_{j}$ at the output of the channel when the input is $\alpha_{i}$. Recall that we assume that the matrix $\Pi$ is known both by the encoder and the decoder.

Let $\lambda: \mathcal{X} \times \hat{\mathcal{X}} \rightarrow \mathbb{R}^{+}$be the loss function (fidelity criterion) which measures the loss incurred in denoising (decoding) a symbol $\alpha_{i}$ to another symbol $\alpha_{j}$, which will be represented by a $N \times N$ matrix, $\boldsymbol{\Lambda}:\left\{\lambda\left(\alpha_{i}, \alpha_{j}\right)\right\}$. Moreover, let

$$
\lambda_{\max }=\max _{i, j} \lambda\left(\alpha_{i}, \alpha_{j}\right)
$$

and note that $\lambda_{\max }<\infty$, since the alphabets are finite. The normalized cumulative loss between a source sequence $x^{n}$ and reconstructed sequence $\hat{x}^{n}$, is denoted by

$$
\rho_{n}\left(x^{n}, \hat{x}^{n}\right)=\frac{1}{n} \sum_{i=1}^{n} \lambda\left(x_{i}, \hat{x}_{i}\right) .
$$

Let $\pi_{i}$ and $\lambda_{j}$ denote the $i$ th column and the $j$ th column of $\Pi$ and $\Lambda$ matrices respectively, i.e.

$$
\boldsymbol{\Pi}=\left[\pi_{1}|\ldots| \pi_{N}\right], \quad \boldsymbol{\Lambda}=\left[\lambda_{1}|\ldots| \lambda_{N}\right] .
$$

For $N$-dimensional vectors $\mathbf{u}$ and $\mathbf{v}, \mathbf{u} \odot \mathbf{v}$ denotes the $N$-dimensional vector that results from componentwise multiplication of $\mathbf{u}$ and $\mathbf{v}$, i.e.

$$
\mathbf{u} \odot \mathbf{v}[i]=u_{i} v_{i}
$$

As in (2), we denote the $i$ th component of a vector by either a subindex or, when that could lead to some confusion, in square brackets.

\section{Dude With Fidelity BoOsting INFORMATION}

The DUDE algorithm was proposed in [12] for universal noncausal denoising of a discrete signal corrupted by a known DMC. The DUDE is described by (3) and (4)

$$
\hat{X}^{n, l}\left(z^{n}\right)[i]=\arg \min _{\hat{x} \in \mathcal{A}} \mathbf{r}^{T}\left(z^{n}, z_{i-l}^{i-1}, z_{i+1}^{i+l}\right) \Pi^{-1}\left[\lambda_{\hat{x}} \odot \pi_{z_{i}}\right]
$$

where, for $\beta \in \mathcal{Z}$

$$
\begin{aligned}
& \mathbf{r}\left(z^{n}, a^{l}, b^{l}\right)[\beta]= \\
& \quad\left|\left\{l+1 \leq i \leq n-l: z_{i-l}^{i-1}=a^{l}, z_{i+1}^{i+l}=b^{l}, z_{i}=\beta\right\}\right| .
\end{aligned}
$$

Remark: Although in (3) it is implicitly assumed that the transition matrix $\Pi$ is a square matrix, this assumption is not necessary. As noted in [12], as long as the rows of $\Pi$ are linearly independent, the results can be generalized to nonsquare matrices by replacing $\Pi^{-1}$ with $\Pi^{T}\left(\boldsymbol{\Pi} \boldsymbol{\Pi}^{T}\right)^{-1}$ in (3). The linear independence of the rows of $\Pi$ requires the channel inputs to be identifiable, i.e., it is not possible to fake the output distribution corresponding to any of them using some input distribution over the other input symbols. This property, which holds for most channels of interest, will be assumed throughout the paper.

In short, DUDE works as follows. In its first pass through the noisy data, it estimates the conditional marginal distributions of the clean data given their noisy observation of $\mathrm{P}_{X_{i} \mid Z^{n}}(\cdot \mid \cdot)$ by first estimating the bidirectional conditional probabilities $\mathrm{P}_{Z_{i} \mid Z^{n} \backslash i}(\cdot \mid \cdot)$ through counting, and then using the invertibility of the DMC. Then in the second pass, it finds $\hat{x}_{i}$ based on these 
estimations. The DUDE denoising algorithm is noncausal and therefore each output depends on the whole noisy sequence. The following optimality results have been shown for DUDE [12].

1) Stochastic setting: the source is assumed to be stationary, and no further constraints are imposed on its distribution. Asymptotically DUDE performs as well as the best denoiser that knows the source distribution provided that the context length $\ell$ grows adequately with the data size.

2) Semistochastic setting: the source is assumed to be an individual sequence, and the only randomness is assumed to originate from the channel. Asymptotically, DUDE performs as well as the optimal denoiser in the class of slidingwindow denoisers for that particular sequence.

Better experimental performance than the DUDE has been achieved by alternative methods to estimate the bidirectional conditional probabilities [22]-[24]. As we discussed in Section I, decoding for the WZ problem can also be considered as a denoising problem where the denoiser, in addition to the noisy signal, has access to a FB sequence designed by the source encoder to be as helpful as possible to the decoder. From this perspective, we are motivated to generalize DUDE so as to handle not only the output of the DMC, but the fidelity boosting information. A desirable feature of such a generalization is the optimality in senses analogous to those described above. A natural way to accomplish this, which we refer to as fbDUDE is described in (5) and (6).

$$
\begin{aligned}
& \hat{X}^{n, l, m}\left(z^{n}, y^{n}\right)[i] \\
& \quad=\arg \min _{\hat{x} \in \mathcal{A}} \mathbf{r}^{T}\left(z^{n}, y^{n}, z_{i-l}^{i-1}, z_{i+1}^{i+l}, y_{i-m}^{i+m}\right) \Pi^{-1}\left[\lambda_{\hat{x}} \odot \pi_{z_{i}}\right]
\end{aligned}
$$

where, for $\beta \in \mathcal{Z}$, and $t=\max \{l, m\}$ as shown in (6) at the bottom of the page.

Note that the counting process is done simultaneously in both the noisy and FB sequences. Although seemingly more involved, the denoising algorithm described in (5) and (6), is simply the DUDE algorithm working on an enlarged context. For the fbDUDE, the context of each symbol in the noisy signal in addition to the conventional DUDE context of noisy neighboring symbols, consists of the same context window of the FB sequence. Note that in contrast to the conventional context of noisy neighboring symbols, in the context window of the fidelity boosting sequence there is no "hole in the middle". It should be noted that our proposed generalization of the DUDE will not be effective with reasonable computational complexity unless the fidelity boosting sequence depends on the original clean signal in a sequential manner, such as a sliding-window. An example of a nonsequential dependence is a fidelity boosting sequence generated by an arbitrary linear block code.
In order to show that the optimality results of [12] carry over to this case, consider a channel $\tilde{\Pi}$, with input $\tilde{x}_{i}=\left(x_{i}, y_{i}\right)$, and output $\tilde{z}_{i}=\left(z_{i}, y_{i}\right)$, where $z_{i}$ is the output of the original channel $\Pi$, when the input is $x_{i}$. Note that this channel does not disturb the second component of its input vector $\left(x_{i}, y_{i}\right)$. As shown in the next result, since the newly defined channel $\tilde{\boldsymbol{\Pi}}$ inherits its invertibility from the original channel $\Pi$, the results of [12] concerning asymptotic optimality of DUDE can be applied to this case as well.

Theorem 3.1: Provided that $t_{n}|\mathcal{X}|^{2 t_{n}}=o(n / \log n), \forall \mathbf{x}, \mathbf{y}$

$$
\begin{aligned}
& \lim _{n \rightarrow \infty}\left[\frac{1}{n-2 t_{n}} \sum_{i=t_{n}+1}^{n-t_{n}} \lambda\left(x_{i}, \hat{x}_{i}\right)\right] \\
&=\lim _{n \rightarrow \infty} D_{l_{n}, m_{n}}\left(x^{n}, y^{n}, Z^{n}\right), \text { a.s. }
\end{aligned}
$$

where

$$
\begin{aligned}
D_{l_{n}}, m_{n} & \left(x^{n}, y^{n}, z^{n}\right) \\
& =\min _{f: \mathcal{Y}^{2 l_{n}+1} \times \mathcal{Z}^{2 m_{n}+1} \rightarrow \mathcal{X}} \frac{\sum_{i=t_{n}+1}^{n-t_{n}} \lambda\left(x_{i}, f\left(y_{i-l_{n}}^{i+l_{n}}, z_{i-m_{n}}^{i+m_{n}}\right)\right)}{n-2 t_{n}}
\end{aligned}
$$

and $\hat{x}_{i}$ is the output of the denoiser in (5) and (6) with parameters $l_{n}, m_{n}$, and $t_{n} \triangleq \max \left\{l_{n}, m_{n}\right\}$.

Remark 1: Here the class of decompressors is restricted to sliding-window decoders of finite-window length on both noisy data and FB sequence. Theorem 3.1 states that in the semistochastic setting where both the source and the FB sequence are individual sequences, with probability one, the asymptotic accumulated loss of the fbDUDE decoder is no more than the loss incurred by the best decoder of the same order in this class.

Remark 2: Although Theorem 3.1 is stated for the semistochastic setting, as in [12], there is a counterpart in the stochastic setting where the source and FB sequences are jointly stationary processes.

\section{SLIDING-WINDOW WyNER-ZIV CODING}

The majority of achievability proofs in the information theory literature are based on the idea of random block coding. Shannon pioneered this technique for proving his coding theorems for both lossy compression and channel coding. In rate-distortion theory, besides the conventional block codes, sliding block codes were introduced in 1975 by Gray, Neuhoff, and Ornstein [11] and independently by Marton [25], and shown to achieve the (block-coding) rate-distortion function in [11]. SB encoders apply a function with a finite number of arguments to the source sequence, outputting another sequence

$$
\mathbf{r}\left(z^{n}, y^{n}, a^{l}, b^{l}, c_{-m}^{m}\right)[\beta]=\left|\left\{t+1 \leq i \leq n-t: z_{i-l}^{i-1}=a^{l}, z_{i+1}^{i+l}=b^{l}, y_{i-m}^{i+m}=c_{-m}^{m}, z_{i}=\beta\right\}\right| .
$$


that has lower entropy, but resembles the original sequence as much as the designer desires.

In the rest of the section, we show that sliding block WZ coding achieves the Wyner-Ziv rate-distortion function for stationary sources.

\section{A. Block Coding}

A WZ block code of length $n$ and rate $R$ consists of encoding and decoding mappings, $f_{n}$ and $g_{n}$, respectively, which are defined as follows:

$$
\begin{aligned}
& \mathrm{f}_{n}: \mathcal{X}^{n} \rightarrow\left\{1,2, \ldots,\left\lceil 2^{n R}\right\rceil\right\} \\
& \mathrm{g}_{n}: \mathcal{Z}^{n} \times\left\{1,2, \ldots,\left\lceil 2^{n R}\right\rceil\right\} \rightarrow \hat{\mathcal{X}}^{n}
\end{aligned}
$$

The performance of such code is defined as the expected average distortion per symbol between the source and reconstruction sequences, i.e.

$$
\mathrm{E}\left[\rho_{n}\left(X^{n}, \hat{X}^{n}\right)\right] \triangleq \frac{1}{n} \mathrm{E}\left[\sum_{i=1}^{n} \lambda\left(X_{i}, \hat{X}_{i}\right)\right]
$$

where $\hat{X}^{n}=\mathrm{g}_{n}\left(Z^{n}, \mathrm{f}_{n}\left(X^{n}\right)\right)$.

The rate distortion pair $(R, D)$ is said to be achievable if for any given $\epsilon>0$, there exists $\mathrm{f}_{n}$, and $\mathrm{g}_{n}$, such that

$$
\mathrm{E}\left[\rho_{n}\left(X^{n}, \mathrm{~g}_{n}\left(Z^{n}, \mathrm{f}_{n}\left(X^{n}\right)\right)\right)\right] \leq D+\epsilon
$$

for all sufficiently large $n$. For a given source $\mathbf{X}$, and memoryless channel described by transition matrix $\Pi$, the infimum of all achievable distortions at rate $R$ is called $D_{\mathbf{X}, \Pi}$, i.e.

$$
D_{\mathbf{X}, \boldsymbol{\Pi}}(R)=\inf \{D:(R, D) \text { is achievable }\}
$$

More explicitly, $D_{\mathbf{X}, \Pi}(R)$ is the distortion-rate function of our WZ coding setting.

\section{B. Sliding-Block WZ Compression}

An extension of the idea of SB rate distortion coding is SB WZ coding. In this section, using the techniques of [11], we show that in WZ coding any performance that is achievable by block codes is also achievable by SB codes.

A WZ SB code consists of two time-invariant encoding and decoding mappings $f$ and $g$. The encoding mapping $f$ with constraint length of $2 k+1$ maps every $2 k+1$ source symbols into a symbol of $\mathcal{Y}$ which is the alphabet of the FB sequence; in other words

$$
f: \mathcal{X}^{2 k+1} \rightarrow \mathcal{Y}
$$

This encoder moves over the source sequence and generates the FB sequence $\mathbf{Y}$ by letting

$$
Y_{i}=f\left(X_{i-k}^{i+k}\right)
$$

On the other hand, the decoding mapping $g$ with the constraint length of $\max \{2 l+1,2 m+1\}$ maps a block of length $2 l+1$ of the noise corrupted signal and a block of length $2 m+1$ of $\mathbf{Y}$ sequence to a reconstruction symbol, i.e.

$$
g: \mathcal{Z}^{2 l+1} \times \mathcal{Y}^{2 m+1} \rightarrow \hat{\mathcal{X}}
$$

The decoder slides over the noisy and the FB sequences in a synchronous manner, and generates the reconstruction sequence by letting

$$
\hat{X}_{i}=g\left(Z_{i-l}^{i+l}, Y_{i-m}^{i+m}\right)
$$

The following theorem states that SB-WZ codes perform at least as well as WZ block codes.

Theorem 4.1: Let $(R, D)$ be an interior point in the (block) WZ rate-distortion region of a jointly stationary processes $\mathbf{X}$ and $\mathbf{Z}$ representing the source and FB sequences respectively. For any given $\epsilon_{1}>0$, there exists a SB-WZ encoder $f: \mathcal{X}^{2 k+1} \rightarrow \mathcal{Y}$, where $\log |\mathcal{Y}| \geq R$, and a SB decoder $g$ with parameters $l$ and $m$, such that

1) $\mathrm{E}\left[\lambda\left(X_{i}, g\left(Z_{i-l}^{i+l}, Y_{i-m}^{i+m}\right)\right)\right] \leq D+\epsilon_{1}$, where $Y_{i}=$ $f\left(X_{i-k}^{i+k}\right)$,

2) $H(\mathbf{Y})=\lim _{n \rightarrow \infty} \frac{1}{n} H\left(Y_{1}, \ldots, Y_{n}\right) \leq R-\epsilon_{2}$, for some $\epsilon_{2}>0$.

Proof: The complete proof is presented in Appendix A; a sketch of the main idea follows. The proof is an extension of the proof given in [11] for showing that SB codes achieve the same performance of block codes in the rate-distortion problem, which in our scenario corresponds to the case where the decoder has only access to the FB sequence and there is no channel output.

Since $(R, D)$ is assumed to be an interior point of the achievable region in the $R-D$ plane, it is possible to find a point $\left(R_{1}, D\right)$ such that $R_{1}<R$, but still the new point is an interior point of the achievable region. Since $\left(R_{1}, D\right)$ is an interior point, there exists a block WZ encoder/decoder, $\left(\mathrm{f}_{n}, \mathrm{~g}_{n}\right)$ of rate $R_{1}$ and block length $n$, and average expected distortion less than $D+\epsilon$, for any $\epsilon>0$. Instead of considering the initial point of $(R, D)$, we consider this new point with $R_{1}<R$ because, according to the theorem, our goal is to show that there exists a SB encoder resulting in a FB sequence with entropy rate lower than $R$. In order to achieve this goal we follow techniques similar to those in [11]. To derive a SB code from a block code, the most challenging part is dividing the source sequence into blocks of fixed length such that it is possible to apply the block code to these sub-blocks. This is a demanding task first because we are looking for a stationary SB code, and second because the decoder is also a SB decoder which should be able to discriminate between different coded blocks concatenated by the encoder. The main tool in our proof, as in [11], is the Rohlin-Kakutani theorem of ergodic theory. This theorem enables us to define a SB encoder which finds blocks of length $n$ to apply the block code to them, and puts a tag sequence of negligible length $\lceil n \epsilon\rceil$ after each encoded block. This tag sequence is not included in any of the codewords of the WZ block 
coder $\left(\mathrm{f}_{n}, \mathrm{~g}_{n}\right)$ (the existence of such tag sequence is shown in the proof). This would enable the decoder to discriminate between different coded blocks, while letting the encoder to generate a stationary FB sequence. The rest of the proof is devoted to showing that the SB code defined in this way would satisfy our desired constraints.

To conclude this section, note that the two-step achievability proof of Wyner and Ziv in [1] for proving their WZ theorem (rate-distortion with side information) is extended in [26] to devise a method which is used to prove a few SB source coding theorems (theorems of Berger, Kaspi and Tung [27]-[29]) for a general finite-alphabet ergodic multiterminal source. The focus in [26] is on multiterminal sources for which we can no longer use the method used in [11] to derive SB codes because the tag sequences in the coded version received by different terminal are not synchronized. In our case, since we have only one terminal to code, and the side information is just the output of the DMC due to the source, it is still possible to use the method used in [11].

\section{WYNER-ZIV DUDE}

In Section III, we introduced the fbDUDE, a natural extension of the DUDE algorithm to the case where in addition to the noisy signal the denoiser has access to encoded side information. As described in Section III, this extension could easily be obtained by considering a larger context for denoising each symbol which comes from working on both signals simultaneously. Then Theorem 3.1 expressed the asymptotic optimality of the fbDUDE denoiser. Section IV introduced SB-WZ coding, and in Theorem 4.1 it was shown that using SB-WZ codes instead of WZ block codes incurs no loss of optimality. Motivated by the results established so far, in this section, we propose a new WZ coding scheme, and prove its asymptotic optimality.

For any given block length $n$, let $\mathrm{f}_{n}^{*}$ and $\mathrm{g}_{n}^{*}$ denote the encoder and the decoder of the scheme, respectively. The scheme has a number of parameters, namely $l_{n}, k_{n}, m_{n}$ and $\delta$, that their meaning is made explicit in the following description of the algorithm.

1) Encoder: For a given source sequence $x^{n}$ define $S\left(x^{n}, k_{n}, R\right)$ to be the set of all SB mappings of window length $2 k_{n}+1$ with the property that their output is a sequence whose Lempel-Ziv compressed version $L Z(\cdot)$ is not longer than $n R$, i.e.

$$
S\left(x^{n}, k_{n}, R\right) \triangleq\left\{f: \mathcal{X}^{2 k_{n}+1} \rightarrow \mathcal{Y}: \frac{1}{n} L Z\left(f\left(x^{n}\right)\right) \leq R\right\}
$$

Note that $f\left(x^{n}\right)$ is assumed to be equal to $y^{n}$, where $y_{i}=$ $f\left(x_{i-k_{n}}^{i+k_{n}}\right)$ for $k_{n}+1 \leq i \leq n-k_{n}$, and $y_{i}=0$ otherwise. For each $f \in S$, and integers $l_{n}$ and $m_{n}$ define

$$
\begin{aligned}
& V\left(f, l_{n}, m_{n}\right) \\
& =\min \mathrm{E}\left[\sum_{i=k_{n}+1}^{n-k_{n}} \lambda\left(x_{i}, g\left(Z_{i-l_{n}}^{i+l_{n}}, y_{i-m_{n}}^{i+m_{n}}\right)\right)\right]
\end{aligned}
$$

where the minimization is over all decoding mappings $g$ : $\mathcal{Z}^{2 l_{n}+1} \times \mathcal{Y}^{2 m_{n}+1} \rightarrow \hat{\mathcal{X}}$. Let $f^{*}\left(l_{n}, m_{n}\right)$ be the mapping in $S$ that minimizes $V\left(f, l_{n}, m_{n}\right)$, i.e.

$$
f^{*}\left(l_{n}, m_{n}\right)=\arg \min _{f \in S} V\left(f, l_{n}, m_{n}\right)
$$

and also let $g^{*}$ be the decoder mapping corresponding to $f^{*}$ that achieves $V\left(f^{*}, l_{n}, m_{n}\right)$.

Then, the FB encoded sequence is the LZ compression of $f_{n}^{*}\left(x^{n}\right)$ which is sent to the decoder.

2) Decoder: Upon obtaining $f_{n}^{*}\left(x^{n}\right)$ with an LZ decompressor, the decoder employs the fbDUDE described in Section III, i.e., the reconstructed signal is $\hat{X}^{n, k_{n}, m_{n}}\left(Z^{n}, f^{*}\left(x^{n}\right)\right)$.

The main result of this paper is the following theorem, which shows that the described WZ-DUDE coding algorithm is asymptotically optimal.

Theorem 5.1: Let $k_{n}, l_{n}$, and $m_{n}$ increase without bound with $n$, but sufficiently slowly that $t_{n}|\mathcal{X}|^{t_{n}}=o(n / \log n)$, where $t_{n}=\max \left\{l_{n}, m_{n}\right\}$. Then, for any $R \geq 0$, and any stationary ergodic source $\mathbf{X}$

$$
\lim _{n \rightarrow \infty} \mathrm{E}\left[\rho_{n}\left(X^{n}, \mathrm{~g}_{n}^{*}\left(Z^{n}, \mathrm{f}_{n}^{*}\left(X^{n}\right)\right)\right)\right]=D_{\mathbf{X}, \mathbf{\Pi}}(R) .
$$

Proof: The full proof can be found in Appendix B. A brief outline of the proof is as follows. The first step is using Theorem 4.1, to find a SB-WZ code with mappings $f$ and $g$ which results in a final expected distortion less that $D+\frac{\epsilon}{2}$, and a FB sequence of entropy rate less than $R-\epsilon_{2}$ ( $\epsilon_{2}$ goes to zero as $\epsilon_{1}$ does). The second step uses the fact that for any stationary ergodic process, the LZ coding algorithm is an asymptotically optimal lossless compression scheme. Therefore, by choosing sufficiently large block length, the difference between the bit per symbol resulting from LZ compression of the FB sequence, and its entropy rate could be made sufficiently small. The third step is using the asymptotic optimality of fbDUDE decoding algorithm which guarantees that by choosing decoding window lengths properly, there is no loss in using fbDUDE decoder instead of any other possible sliding-window decoder.

Note that the only part of our scheme of questionable practicality is its encoding which requires listing all mappings of some finite window length which generate a FB sequence with LZ description length less than some fixed value depending on block length and coding rate. This is a huge number, e.g., for the binary case there are $2^{2^{2 k+1}}$ mappings having a window length of $2 k+1$ (for $k=1$ there are 256 mappings). Therefore, we cannot implement the algorithm as described, but as shown in Section IV, this new scheme inspires pragmatic universal WZ coding schemes attaining good performance.

Finally, it is worth mentioning the relationship between our encoding algorithm and the Yang-Kieffer fixed-rate lossy coding algorithm described in [30]: For block length $n$, the encoder constructs a codebook $C_{n}$ consisting of all reconstruction blocks having LZ description length less than $n R$. The $x^{n}$ is represented by the nearest codeword $\hat{x}^{n}$ in $C_{n}$. Yang and Kieffer [30] show that for any stationary ergodic source, this 


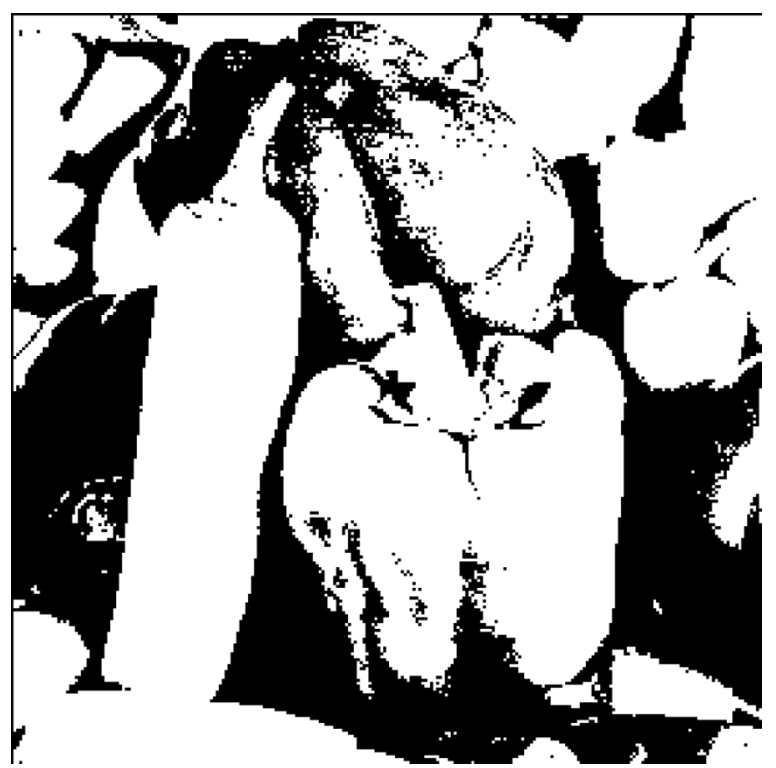

Fig. 2. Original binary image 0.6 b.p.p.

conceptually simple (but not implementable) scheme achieves the rate-distortion function as $n$ goes to infinity. In our case, we construct our codebook in a similar way, but since the encoder knows that the decoder has access to the output of the DMC as well, the codeword that results in minimum average expected loss is chosen (the expectation is taken over all possible channel outputs for the best possible SB decoder).

\section{PRAGMATIC APPROACHES AND EXPERIMENTAL RESUlTS}

As aforementioned, the demanding aspect of the WZ-DUDE algorithm is finding the optimal mapping $f^{*}$. In all of the following cases, instead of looking for the optimal mapping, we choose a not-necessarily optimal mapping along with the WZ-DUDE decoder. Furthermore, in all of the following cases, the distortion measure is Hammig distortion, i.e., for $x, \hat{x} \in \mathcal{X}$

$$
\lambda(x, \hat{x})= \begin{cases}0, & x=\hat{x} \\ 1, & x \neq \hat{x}\end{cases}
$$

\section{A. Binary Image With BSC}

In this experiment, instead of looking for the optimal mapping, we use a lossy JPEG encoder. Since except for the encoding of the DC component, JPEG works on $8 \times 8$ blocks separately, it can be considered as a SB encoder of window length 1 working on the superalphabets formed by $8 \times 8$ binary blocks. Figs. 2 and 3 show the original binary image and its noise-corrupted version under a binary symmetric channel with crossover probability 0.15 . Fig. 4 shows the JPEG encoded image which requires 0.22 bit per pixel (b.p.p.) after JPEG lossless compression, compared to 0.6 b.p.p. required by the original image. The average Hamming distortion between the original image and the decompressed one is 0.0556 . Fig. 5 shows the result of denoising the noise corrupted image with DUDE ignoring the FB sequence. In this case the resulting average distortion is 0.0635 .

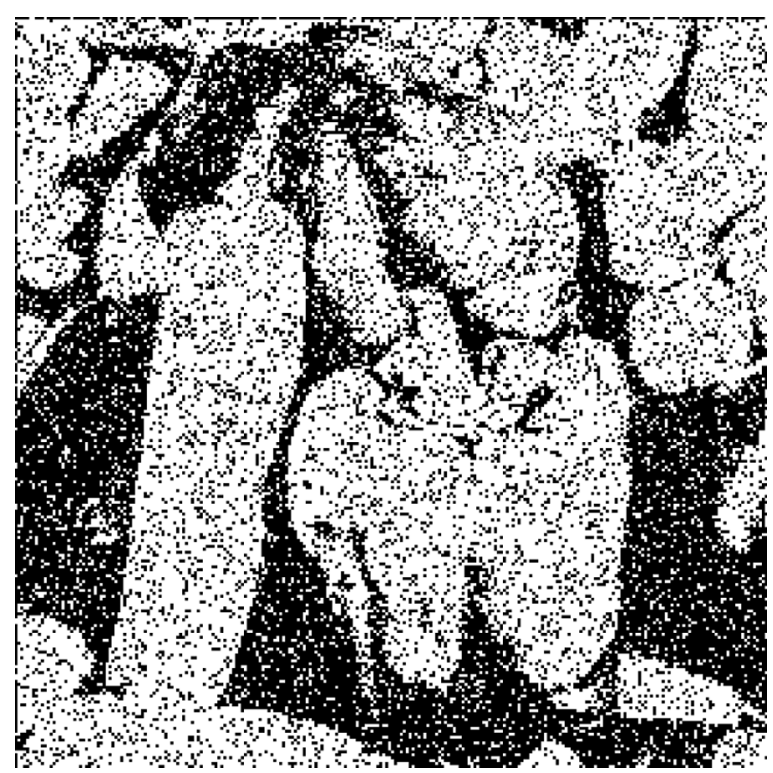

Fig. 3. Noise corrupted image, generated by passing the original signal through a $\operatorname{BSC}(0.15)$.

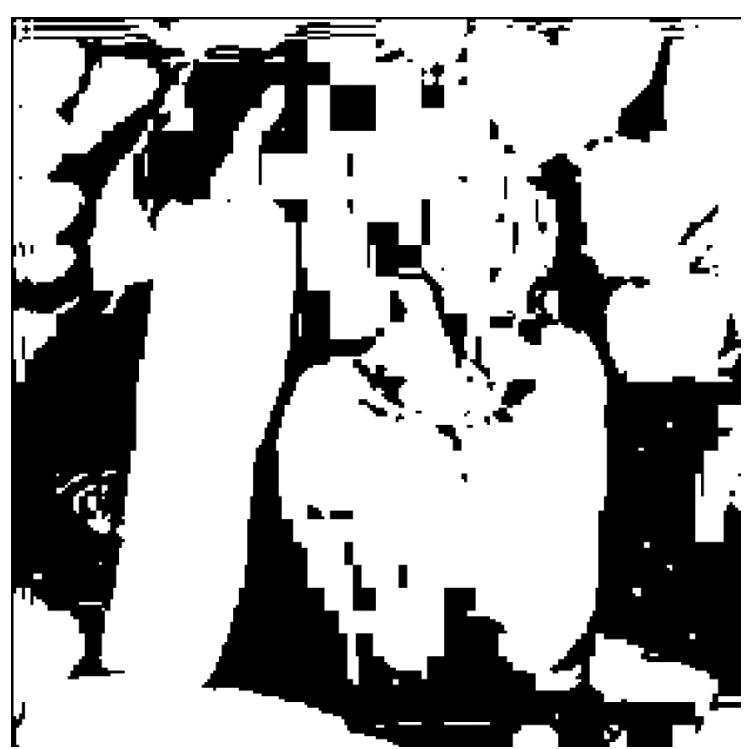

Fig. 4. The FB image, $\mathbf{y}$, generated by lossy JPEG coding of the original image, 0.22 b.p.p., $\rho\left(x^{n}, y^{n}\right)=0.0556$.

On the other hand, Fig. 6 shows the result of denoising the noisy signal when the FB sequence is also taken into account. The decoder/denoiser in this case is WZ-DUDE with parameters $l=1$ and $m=1$. The final average distortion between the reconstructed image and the original image is 0.0407 .

\section{B. Text With Erasure Channel}

In this section, we consider the case where our source is an English text document, and the DMC is a memoryless erasure channel that erases each symbol with probability e. To construct the FB sequence, we use a method which is similar to the first run of the DUDE algorithm in which it tries to estimate $\mathrm{P}_{Z_{i} \mid Z^{n \backslash i}}(\cdot \mid \cdot)$, to estimate $\mathrm{P}_{X_{i} \mid X^{n \backslash i}}(\cdot \mid \cdot)$. For a given window 


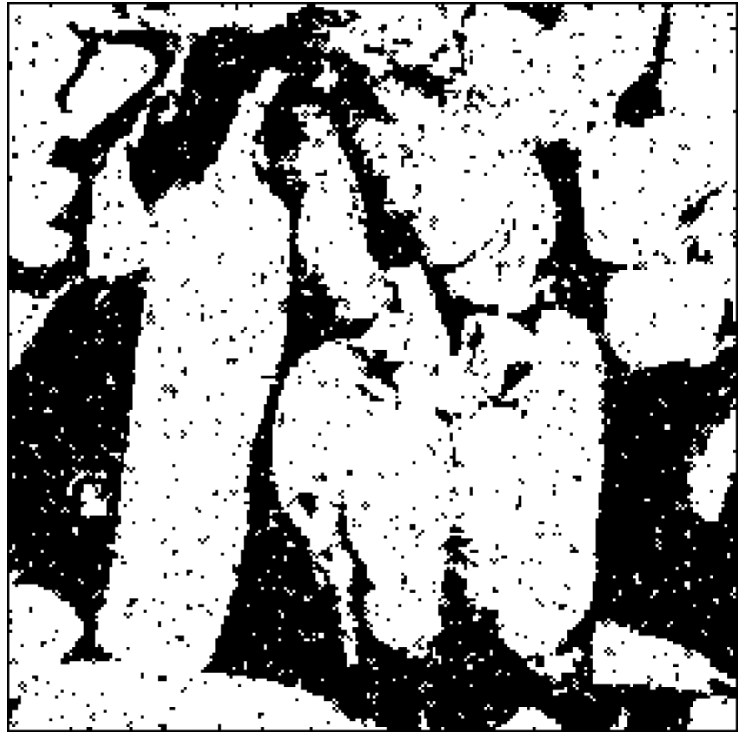

Fig. 5. Output of DUDE for $l=1 . \rho_{n}\left(x^{n}, \hat{x}^{n}\right)=0.0635$.

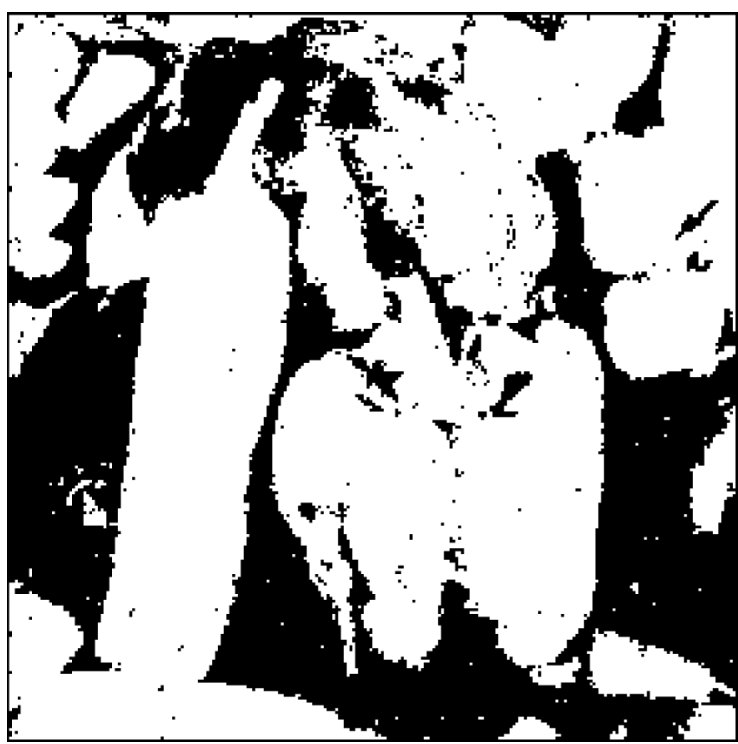

Fig. 6. Output of the WZ-DUDE decoder for $l=m=1$, and $R=0.22$ b.p.p., $\rho_{n}\left(x^{n}, \hat{x}^{n}\right)=0.0407$.

length of $2 k+1$, the encoder generates the count matrix $\mathbf{r}_{\mathrm{enc}}$ as follows:

$$
\mathbf{r}_{\mathrm{enc}}\left(x^{n}, a^{k}, b^{k}\right)[\beta]=\left|\left\{i: x_{i-k}^{i-1}=a^{k}, x_{i+1}^{i+k}=b^{k}, x_{i}=\beta\right\}\right|
$$

For each left and right contexts $a^{k}$ and $b^{k}$, the vector $\mathbf{r}_{\text {enc }}\left(x^{n}, a^{k}, b^{k}\right)$ is a $1 \times|\mathcal{X}|$ vector with $\beta$ th component being equal to the number of times the $\beta$ th element of $\mathcal{X}$ have appeared in $x^{n}$ sequence with its right and left contexts being equal to $a^{k}$ and $b^{k}$ respectively. Therefore, from the count vector $\mathbf{r}_{\text {enc }}\left(x^{n}, x_{i-k}^{i-1}, x_{i+1}^{i+k}\right)$ corresponding to the right and left contexts of $x_{i}$, the MAP estimation of $x_{i}$ is

$$
\underset{\beta \in \mathcal{X}}{\arg \max } \mathbf{r}_{\mathrm{enc}}\left(x^{n}, x_{i-k}^{i-1}, x_{i+1}^{i+k}\right)[\beta]
$$

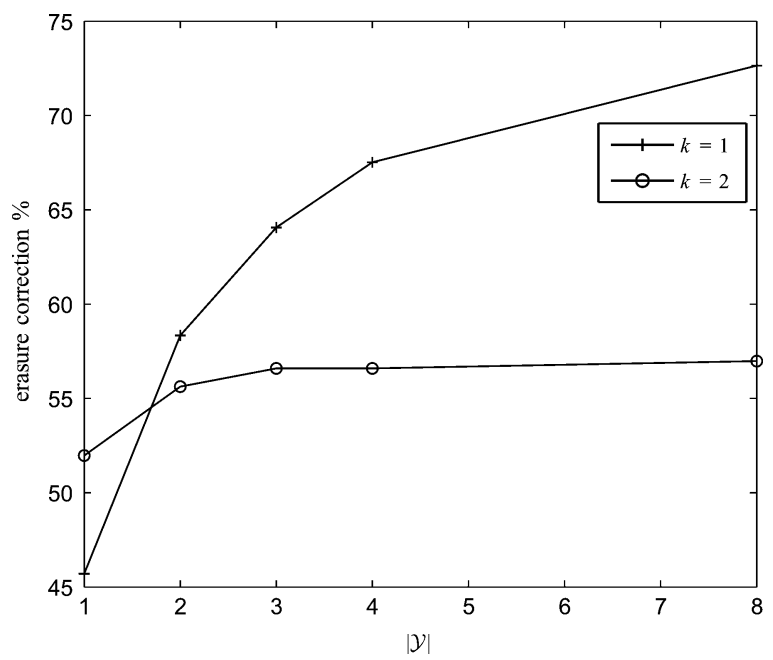

Fig. 7. Percentage of erasures that are recovered by WZ-DUDE decoder versus the size of the FB alphabet $\mathcal{Y}$, for $k=1$ and $k=2$, and $\mathrm{e}=0.1$.

which is the symbol in $\mathcal{X}$ is the most frequent symbol in $x^{n}$ among those with the same right and left contexts of $z_{i}$. Similarly, for given right and left contexts, we can rank all the symbols in $\mathcal{X}$ according to their repetition frequency in our text within the given contexts. Now for a FB alphabet cardinality of $N$, define $\mathcal{Y}=\{1, \ldots, N\}$. The encoding function $f$ is as follows:

$$
f\left(x_{i-k}^{i+k}\right)=\left\{\begin{aligned}
\ell, & \text { if } x_{i}=\beta, \text { where } \mathbf{r}_{\text {enc }}\left(x^{n}, x_{i-k}^{i-1}, x_{i+1}^{i+k}\right)[\beta] \\
& \text { is the } \ell \text { th largest element, and } \ell<N \\
N, & \text { otherwise. }
\end{aligned}\right.
$$

After constructing the sequence $y^{n}$ by sliding $f$ over the original text, the $\mathrm{LZ}$ description of the resulting sequence is transmitted to the decoder. As mentioned in [12], the DUDE denoising rule for an erasure channel is equivalent to a majority-vote of the context counts, i.e., replacing each erasure with the most frequent symbol with the same context. WZ-DUDE decodes the erased symbol $x_{i}$ by first computing $\mathbf{r}_{\mathrm{dec}}\left(z^{n}, z_{i-k}^{i-1}, z_{i+1}^{i+k}\right)$. For moderate values of e, one would expect $\mathbf{r}_{\text {enc }}$, and $\mathbf{r}_{\text {dec }}$ to rank the symbols similarly. Therefore, based on $\mathbf{r}_{\mathrm{dec}}$ count vector, and $y_{i}, \hat{x}_{i}$ is the source alphabet corresponding to the $y_{i}$ th largest element of $\mathbf{r}_{\mathrm{dec}}\left(z^{n}, z_{i-k}^{i-1}, z_{i+1}^{i+k}\right)$. Note that in this case, the window length of the SB encoder and decoder should be the same, otherwise $y^{n}$ does not help the decoder.

Fig. 7 shows the percentage of erased symbols that are recovered by our WZ-DUDE decoder for different values of $N$. For our experiments we have used the English translation of Don Quixote de La Mancha, by Miguel de Cervantes Saavedra $(1547-1616)^{1}$. The text consists of approximately $2.3 \times 10^{6}$ characters. The channel is assumed to have erasure probability 0.1. In Fig. 7, $N=1$, corresponds to the case where there is no FB available to the decoder, or in other words, it corresponds to the performance of the DUDE. As it can observed, for $N=1$ using larger context size $k$ improves performance; As $N$ increases, $k=1$ outperforms $k=2$. In addition both curves seem

${ }^{1}$ which is available online from the Project Gutenberg web-site at http://promo.net/pg/http://promo.net/pg/ 


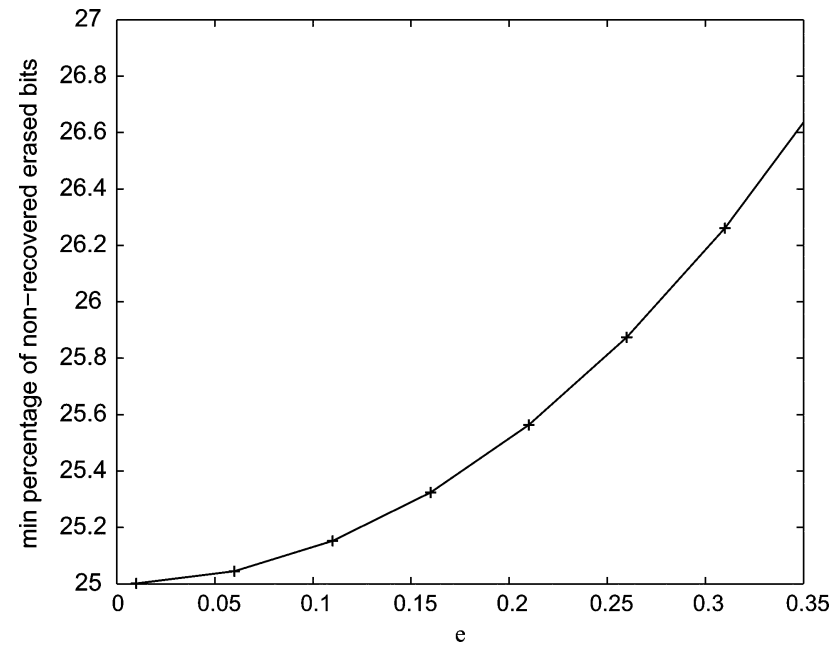

Fig. 8. $\quad \frac{100}{e} \mathrm{P}\left(\hat{X}_{i} \neq X_{i}\right)$ versus e, where $\mathrm{P}\left(\hat{X}_{i} \neq X_{i}\right)$ is the probability of error of the optimal denoiser that knows the source distribution, and is computed using (20), for a BSMS with $q=0.25$ passing through an erasure channel which erasure probability of $\mathrm{e}$.

to eventually saturate as $N$ increases. Although one might expect that increasing $N$ would always improve the performance, and tending it to $|\mathcal{X}|$, one should be able to recover all erased symbols, we see in Fig. 8, this does not hold for our scheme. The reason is that, once one of the symbols in the context of an erased symbol is erased, the decoder is not able to construct the count vector $\mathbf{r}_{\mathrm{dec}}\left(z^{n}, z_{i-k}^{i-1}, z_{i+1}^{i+k}\right)$, which is crucial in interpreting the FB sequence. In such cases we let $\hat{x}_{i}$ to be the space character which has the largest frequency in the text. Therefore, the best error-correction performance that can be achieved by our scheme is upper bounded by the probability that none of the symbols in the context of an erased symbol are erased, which is equal to $(1-\mathrm{e})^{2 k}$. In our example, for $k=1$ the upper bound is $0.9^{2}=0.81$, and for $k=2$, it is $0.9^{4} \approx 0.66$, which coincides with our curves.

To illustrate the performance of the algorithm, a small excerpt of length 154 of the original text, its noise-corrupted version, and the outputs of its DUDE and WZ-DUDE decoded versions, for different values of $k$, and $N$ are presented.

\section{- Clean text:}

... methodising with rare patience and judgment what had been previously brought to light, he left, as the saying is, no stone unturned under which anything,...
- Erasure-corrupted source: (12 erasures)

... *et*odising with ra*e pati*nce and judgment what had been previously brought to ligh*, he $1^{*} \mathrm{ft}^{*}$ as the $\mathrm{s}^{*} \mathrm{yin} *$ is, no stone untu*ned under which any*hin*,...

- DUDE denoiser with no FB sequence, $k=1$ (7 errors + 1 erasure)

... bethodising with rave pationce and judgment what had been previously brought to ligho, he lifto as the sayind is, no stone unturned under which any*hing, ...

- WZ-DUDE denoiser, $k=1, N=2, R=0.16$ b.p.s. ( 2 errors)

... mhethodising with rare patience and judgment what had been previously brought to light, he lefth as the saying is, no stone unturned under which anything,...

- DUDE denoiserwith no FB, $k=2$ ( 3 errors)

$\ldots$ bethodising with rate patience and judgment what had been previously brought to light, he lefts as the saying is, no stone unturned under which anything,,..

- WZ-DUDE denoiser, $k=2, N=2, R=0.12$ b.p.s. ( 2 errors)

... rethodising with race patience and judgment what had been previously brought to light, he left, as the saying is, no stone unturned under which anything,...

\section{Binary Markov Source With Binary Erasure Channel}

Consider a binary symmetric Markov source with transition probability $q$, denoted by $B S M S(q)$, which goes through a memoryless binary erasure channel (BEC) with erasure probability e. Let $X^{n}$ and $Z^{n}$ be the input and output of the channel respectively. Note that in this case $\mathcal{X}=\{0,1\}$, and $\mathcal{Z}=\{0,1, e\}$, where $e$ denotes an erased symbol. Without having access to any other information, the optimal denoising rule which minimizes the probability of error is $\hat{X}_{i}=\underset{\alpha \in \mathcal{X}}{\arg \max } \mathrm{P}\left(X_{i}=\alpha \mid Z^{n}\right)$. The probability of error of this optimal denoiser is shown in (20) at the bottom of the page, where

$$
\begin{aligned}
& f(l, r, \alpha, \beta) \\
& \triangleq \mathrm{P}\left(\hat{X}_{i} \neq X_{i}, Z_{i-l+1}^{i+r-1}=\mathrm{e}_{r+l-1}, Z_{i-l}=\alpha, Z_{i+r}=\beta\right) \\
& =\min \left\{\mathrm{P}\left(X_{i}=0, Z_{i-l+1}^{i+r-1}=\mathrm{e}_{r+l-1}, Z_{i-l}=\alpha, Z_{i+r}=\beta\right)\right. \\
& \left.\quad \mathrm{P}\left(X_{i}=1, Z_{i-l+1}^{i+r-1}=\mathrm{e}_{r+l-1}, Z_{i-l}=\alpha, Z_{i+r}=\beta\right)\right\}
\end{aligned}
$$

$$
\begin{aligned}
\mathrm{P}\left(\hat{X}_{i} \neq X_{i}\right)= & \mathrm{P}\left(\hat{X}_{i} \neq X_{i}, Z_{i} \neq e\right) \\
& +\sum_{l=1}^{\infty} \sum_{r=1}^{\infty} \mathrm{P}\left(\hat{X}_{i} \neq X_{i}, Z_{i-l} \neq e, Z_{i+r} \neq e Z_{j}=e, \text { for } j=i-l+1, \ldots, i+r-1\right) \\
= & \sum_{l=1}^{\infty} \sum_{r=1}^{\infty} \sum_{\alpha \in \mathcal{X}} \sum_{\beta \in \mathcal{X}} f(l, r, \alpha, \beta)
\end{aligned}
$$


where $\mathrm{e}_{m}$ denotes a vector of length $m$ with all elements equal to $e$. Note that $f(l, m, \alpha, \beta)$ is an easily computable function. For example, for $q<\frac{1}{2}$

$$
\begin{aligned}
f(1,1,0,0) & =f(1,1,1,1), \\
& =\mathrm{P}\left(\hat{X}_{i} \neq X_{i}, Z_{i}=e, Z_{i-1}=Z_{i+1}=1\right) \\
& =\frac{q^{2}}{2} \mathrm{e}(1-\mathrm{e})^{2}
\end{aligned}
$$

and shown in (23)-(27) at the bottom of the page.

Similar expressions can be derived for higher values of $l$ and $m$. Note that

$$
\lim _{\mathrm{e} \rightarrow 0} \frac{1}{\mathrm{e}} \mathrm{P}\left(\hat{X}_{i} \neq X_{i}\right)=q
$$

and

$$
\lim _{\mathrm{e} \rightarrow 1} \frac{1}{\mathrm{e}} \mathrm{P}\left(\hat{X}_{i} \neq X_{i}\right)=0.5 \text {. }
$$

Fig. 8 shows the percentage of erased symbols decoded erroneously versus e, for a BSMS with $q=0.25$. The points in Fig. 8 are computed by evaluating (20), and, therefore, reflect the performance of an optimal denoiser that knows the source distribution. It can be observed that as e increases this percentage increases as well. The reason is that for small values of e each erased symbol is surrounded by nonerased symbols with high probability, and therefore since $q$ is relatively small, the erased symbol can be recovered correctly with high probability. On the other hand, as e increases, the probability of having two consecutive erased symbols, which are harder to recover, increases as well.

Now consider the WZ setup, where in addition to the output of the BEC, the decoder has access to a FB sequence of rate $R$ designed by the encoder to improve the decoder's performance. For generating this FB sequence we again use DUDE counts.
For a fixed $k$, the encoder first forms the count matrix consisting of $\mathbf{r}_{\text {enc }}\left(a^{k}, b^{k}\right)$ for all $2^{2 k}$ possible right and left contexts, each of length $k$. Then the FB sequence is

$$
Y_{i}=\left\{\begin{array}{cc}
0, & \text { if } \mathbf{r}_{\text {enc }}\left(X_{i-k}^{i-1}, X_{i+1}^{i+k}\right)\left[X_{i}\right] \geq \\
& \mathbf{r}_{\text {enc }}\left(X_{i-k}^{i-1}, X_{i+1}^{i+k}\right)\left[1-X_{i}\right] \\
1, & \text { otherwise }
\end{array}\right.
$$

In other words, $Y_{i}$ is 1 whenever $X_{i}$ is different from what it is predicted to be from its context. As aforementioned, the DUDE decision rule for the BEC is majority-vote decoding. In the case of a binary source instead of text, if there are erased symbols in the context of an erased bit, we do not simply let $\hat{X}_{i}$ equal to some prefixed symbol. When in addition to $Z_{i}$ some other bits of $Z_{i-k}^{i+k}$ are erased, the decoder's count vector $\mathbf{r}_{\mathrm{dec}}\left(Z_{i-k}^{i-1}, Z_{i+1}^{i+k}\right)$ is the average of count vectors corresponding to all possible binary contexts coinciding with $Z_{i-k}^{i+k}$ at the nonerased positions. If $k_{e}$ bits out of $2 k$ are erased, then there exist $2^{k_{e}}$ such contexts that agree with original context in the $2 k-k_{e}$ nonerased bits. Let $b=\underset{\beta \in\{0,1\}}{\arg \max } \mathbf{r}_{\mathrm{dec}}\left(Z_{i-k}^{i-1}, Z_{i+1}^{i+k}\right)[\beta]$, then

$$
\hat{X}_{i}= \begin{cases}b, & \text { if } \quad Y_{i}=0 ; \\ 1-b, & \text { if } \quad Y_{i}=1 .\end{cases}
$$

Consider again the BSMS with $q=0.25$ passed through a BEC with $e=0.1$. From Fig. 8, an optimal denoiser that only has access to the output of BEC will decode at least $25.13 \%$ of the erased bits wrongly. On the other hand, the DUDE denoiser decodes $25.44 \%$ of erased bits erroneously, which is almost equal to the performance of the optimal nonuniversal denoiser which knows the statistics of the source. Now assume that the encoder also sends to the decoder the FB sequence $Y^{n}$ constructed as described in (30). From our simulation results, for this case, the entropy of the FB sequence is around $R=0.3$, and applying the described WZ-DUDE decoder to $\left(Y^{n}, Z^{n}\right)$ reduces the probability of error to $19.3 \%$.

$$
\begin{aligned}
f(1,1,0,1)= & f(1,1,1,0) \\
= & \mathrm{P}\left(\hat{X}_{i} \neq X_{i}, Z_{i}=e, Z_{i-1}=1-Z_{i+1}=1\right) \\
= & \frac{1}{2} q(1-q) \mathrm{e}(1-\mathrm{e})^{2}, \\
f(1,2,0,0)= & f(1,2,1,1)=f(2,1,1,1)=f(2,1,0,0) \\
= & \mathrm{P}\left(\hat{X}_{i} \neq X_{i}, Z_{i}=Z_{i+1}=e, Z_{i-1}=Z_{i+2}=1\right) \\
= & q^{2}(1-q) \mathrm{e}^{2}(1-\mathrm{e})^{2}, \\
f(1,2,0,1)= & f(1,2,1,0)=f(2,1,0,1)=f(2,1,1,0) \\
= & \mathrm{P}\left(\hat{X}_{i} \neq X_{i}, Z_{i}=Z_{i+1}=e, Z_{i-1}=1-Z_{i+2}=1\right) \\
= & \frac{1}{2}\left(q^{3}+(1-q)^{2} q\right) \mathrm{e}^{2}(1-\mathrm{e})^{2}, \\
f(2,2,0,0)= & f(2,2,1,1) \\
= & \mathrm{P}\left(\hat{X}_{i} \neq X_{i}, Z_{i-1}=Z_{i}=Z_{i+1}=e, Z_{i-2}=Z_{i+2}=1\right) \\
= & 2 q^{2}(1-q)^{2} \mathrm{e}^{3}(1-\mathrm{e})^{2}, \\
f(2,2,0,1)= & f(2,2,1,0) \\
& \mathrm{P}\left(\hat{X}_{i} \neq X_{i}, Z_{i-1}=Z_{i}=Z_{i+1}=e, Z_{i-2}=1-Z_{i+2}=1\right) \\
= & \frac{1}{2}\left(2 q(1-q)^{3}+2 q^{3}(1-q)\right) \mathrm{e}^{3}(1-\mathrm{e})^{2}
\end{aligned}
$$




\section{CONCLUSION AND FUtURE DiRECTIONS}

This paper deals with WZ coding of a source with unknown statistics; a new WZ coding algorithm, WZ-DUDE, was presented and its asymptotic optimality was established. In order to optimize the scheme one would list all possible mappings that have a certain property and look for the one that gives minimum expected loss. However, we saw that even a simple encoding mapping, namely an off-the-shelf lossy compressor, achieves considerable improvement compared to the case where either the FB sequence or the noisy signal are not present at the decoder.

The original DUDE is tailored to discrete-alphabet sources going through a DMC, and making it applicable to continuous alphabet sources entails more than a trivial extension, which has been accomplished in [31] and [32]. As mentioned in Section III, since our fbDUDE decoder is a special case of the original DUDE algorithm, one would expect that by following the same methods used in [31], [32], it might be possible to devise a decoder which works on continuous data. The nontrivial part is finding a proper encoder. In this case it is not possible to list all SB encoders of some finite block length, because there are an infinite number of them even for window length of one. One simple solution is to look into all mappings which map to a quantized version of the source alphabet. How to choose this quantized alphabet, and whether this would result in a scheme that asymptotically achieves the performance bounds is a question that requires further study. Finding a sequential version of the described scheme, where the decoder is subject to a delay constraint is another interesting open avenue. Adapting our WZ-DUDE algorithm to perform effectively with nonstationary data is another open avenuee. For example, often real data is more accurately modeled as a piecewise stationary source. In the recent work [33], the sDUDE denoising algorithm is described which, unlike DUDE, tries to compete with a genie-aided SB denoiser that can switch between SB denoisers up to $m$ times, where $m$ is sublinear in the block length $n$. When the clean data is emitted by a piecewise stationary process, the sDUDE algorithm achieves the optimum distributiondependent performance.

\section{APPENDIX A}

\section{PROOF OF THEOREM 4.1}

The proof is an extension of the proof given in [34] which is for the case where there is no FB sequence. Let $\mathbf{X}=\left\{X_{i} ; \forall i \in\right.$ $\left.\mathbb{N}^{+}\right\}$be a stochastic process defined on a probability space $(\mathbf{X}, \Sigma, \mathrm{P})$, where $\Sigma$ denotes the $\sigma$-algebra generated by cylinder sets, and $\mathrm{P}$ is a probability measure defined on it. The shift operator $T: \mathcal{X}^{\infty} \rightarrow \mathcal{X}^{\infty}$ is defined by

$$
(T \mathbf{x})_{n}=x_{n+1}, \quad \mathbf{x} \in \mathcal{X}^{\infty}, n \geq 1
$$

Let $\mathcal{X}$ and $\hat{\mathcal{X}}$ denote the source and reconstruction alphabets, respectively, which are both assumed to be finite.

Since $(R, D)$ is assumed to be an interior point of the achievable region in the $R-D$ plane, there exists $\delta_{0}>0$, such that $(R-\delta, D)$ is also an interior point for any $0<\delta \leq \delta_{0}$. Define $R_{1} \triangleq R-\delta$. Since $\left(R_{1}, D\right)$ is an achievable point, for any given $\epsilon>0$, there exists a block WZ encoder/decoder, $\left(\mathrm{f}_{n}, \mathrm{~g}_{n}\right)$ of rate $R_{1}$ and block length $n$, which is sufficiently large based on $\epsilon$, and average expected distortion less than $D+\epsilon$. Assume that among our infinite choices, we pick a WZ code whose block length $n$ is large enough such that

$$
\max \left\{\frac{1}{\sqrt{n}}, \frac{\log n}{n \log |\mathcal{Y}|}\right\}<\epsilon .
$$

This constraint will be useful in our future analysis.

In order to prove that there exists a SB code satisfying the constraints given in Theorem 4.1 , the given block code $\left(f_{n}, g_{n}\right)$ should somehow be embedded in the SB encoder/decoder mappings. For defining a SB code based on a block code, the natural question is how to define blocks in the infinite length source sequence. Moreover, after finding a way for distinguishing blocks in the input sequence, the next problem is how the decoder is going to detect the coded blocks in the infinite length received FB sequence. To answer the first question, as usually done in the literature, we resort to the Rohlin-Kakutani (RK) Theorem of ergodic theory [35].

Theorem 7.1 (Rohlin-Kakutani Theorem): Given the ergodic source $[A, \mu, U]$, integers $L$ and $n \leq L$, and $\epsilon>0$, there exists an event $F$ (called the base ) such that

1) $F, T F, \ldots, T^{L-1} F$ are disjoint,

2) $\mathrm{P}\left(\bigcup_{i=0}^{L-1} T^{i} F\right) \geq 1-\epsilon$,

3) $\quad \mathrm{P}\left(\mathcal{S}\left(a^{n}\right) \mid F\right)=\mathrm{P}\left(\mathcal{S}\left(a^{n}\right)\right)$

4) $\mathcal{S}\left(a^{n}\right)=\left\{\mathrm{x}: x^{n}=a^{n}\right\}$.

This theorem states that for any given $L$, and any $n$ less than $L$, there exists a base event $F$, such that the base and its $L$ disjoint shifts, basically cover the event space, i.e., any given sequence $\mathrm{X}$ with high probability belongs to $T^{i} F$ for some $0 \leq i \leq L-1$. The last property states that the probability distribution of the $n$-tuples is the same both in the base and in the whole space.

For a given $\epsilon>0, n$, the block length of the block encoder/de$\operatorname{coder}\left(\mathrm{f}_{n}, \mathrm{~g}_{n}\right)$, and $L_{n} \triangleq n+\lceil n \epsilon\rceil$, let $F$ be the base event given by the RK theorem for these parameters. Define $G$ to be everything in the event space which is not included in $\bigcup_{i=0}^{L_{n}-1} T^{i} F$. Note that by the RK theorem $\mathrm{P}(G) \leq \epsilon$. To show the existence of a finite length SB encoder, we first prove the existence of an infinite length SB encoder, $f^{(\infty)}$, and then show that it can be truncated appropriately such that the resulting finite window length code also satisfies our desired properties.

Note that $f^{(\infty)}$ maps every infinite length sequence $\mathbf{x}$ into a symbol in the FB sequence alphabet $\mathcal{Y}$, and defines the FB sequence as $\hat{y}_{i}=f^{(\infty)}\left(T^{i} \mathbf{x}\right)$. As mentioned earlier, one problem is enabling the decoder to discriminate between the encoded blocks embedded in the FB sequence. One simple solution is requiring the SB encoder to interject a predefined synchronization sequence, which is not contained in any of the codewords, between the encoded blocks. Let $\mathbf{s}=\mathbf{1}_{\lceil n \epsilon\rceil}$, where $\mathbf{1}_{r}$ is a vector of length $r$ with all of its elements equal to 1 , denote the synchronization block. From now on, symbols 0 and 1 denote two arbitrary distinct symbols in $\mathcal{Y}$. The following lemma shows that as long as $|\mathcal{Y}|>2^{R-\epsilon_{n}}$, it is possible to construct a codebook of $2^{n R}$ distinct codewords none of them containing $\mathbf{s}$. 
Lemma 7.2: If $|\mathcal{Y}|>2^{R-\epsilon_{n}}$, where $\epsilon_{n}=\frac{\log \left(1-n|\mathcal{Y}|^{-\lceil n \epsilon\rceil}\right)}{n}$, it is possible to find a codebook $\mathcal{C} \subset \mathcal{Y}^{n}$ with $2^{n R}$ codewords such that $s=1_{r}$ is not contained in any of them.

Proof: Let $N_{s}$ denote the number of sequences in $\mathcal{Y}^{n}$ that contain $\mathbf{s}$ as part of them. There are $n-\lceil n \epsilon\rceil+1$ positions that might be the start of the $\mathbf{s}$. For each of them it is possible to construct $|\mathcal{Y}|^{n-\lceil n \epsilon\rceil}$ sequences that contain $\mathbf{s}$ starting at that certain position. Therefore, $N_{s}$ is upper-bounded as follows:

$$
N_{s}<(n-\lceil n \epsilon\rceil+1)|\mathcal{Y}|^{n-\lceil n \epsilon\rceil} .
$$

On the other hand, if $|\mathcal{Y}|^{n}-N_{s}>2^{n R}$, then it is possible to choose $2^{n R}$ codewords as desired. Combining this with (A2), it is sufficient to have

$$
|\mathcal{Y}|^{n}-2^{n R}>n|\mathcal{Y}|^{n-\lceil n \epsilon\rceil}
$$

or

$$
|\mathcal{Y}|^{n}\left(1-n|\mathcal{Y}|^{-\lceil n \epsilon\rceil}\right)>2^{n R}
$$

or

$$
\log |\mathcal{Y}|>R-\epsilon_{n}
$$

where $\epsilon_{n}$ is as defined in the statement of the lemma.

Therefore, using the previous lemma, it is possible to construct a codebook $\tilde{\mathcal{C}}$ with $2^{n R_{1}}$ codewords, such that none of them contain s. Further, we can assume that the codewords in $\tilde{\mathcal{C}}$ are chosen such that the first and the last symbols of all of them are equal to 0 . Note that if $|\mathcal{Y}|$ satisfies (A5), then the number of codewords that satisfy the requirement of Lemma 7.2 is exponentially more than $2^{n R}$; Therefore, ir is possible to choose such a codebook. This assumption makes sure that for any $y^{n} \in$ $\mathcal{C}$, the synchronization sequence can uniquely be detected in $y_{1}, \ldots, y_{n}, s_{1}, \ldots, s_{\lceil n \epsilon\rceil}$ and also in $s_{1}, \ldots, s_{\lceil n \epsilon\rceil}, y_{1}, \ldots, y_{n}$ with no ambiguity.

Now each codeword in the codebook $\mathcal{C}$ can be mapped into a unique codeword in $\tilde{\mathcal{C}}$. The role of each element in $\mathcal{C}$ in the coding is then played by the corresponding vector in $\tilde{\mathcal{C}}$ that it is mapped to. Since in the WZ coding, the codebook is only an indexing of the input blocks, such a mapping only acts as a renaming of the vectors in the codebook, and does not have any other effect.

Now we define an infinite length encoder $f^{(\infty)}$ based on the partitioning of the event space given by the RK Theorem as follows:

1) $\mathrm{x} \in T^{i} F$, for some $0 \leq i \leq n-1$ : let $f^{(\infty)}(\mathrm{x})=\hat{y}_{i}$ where $\left[\hat{y}_{0}, \hat{y}_{1}, \ldots, \hat{y}_{n-1}\right] \triangleq \mathrm{f}_{n}\left(x_{-i}^{n-i-1}\right)$.

2) $\mathrm{x} \in T^{i} F$, for some $n \leq i \leq L_{n}-1$ : let $f^{(\infty)}(\mathbf{x})=$ $\mathbf{s}[i-n+1]$

3) $\mathbf{x} \in G$ : let $f^{(\infty)}(\mathbf{x})=y_{0}$, where $y_{0}$ is an element in $\mathcal{Y}$ which is not used in $\mathbf{s}$.

After defining the SB encoder, we can define the SB decoder, $g$, which generates the reconstruction process as $\hat{X}_{i}=$ $g\left(Z_{i-M}^{i+M}, Y_{i-M}^{i+M}\right)$ with $M=2(n+\lceil n \epsilon\rceil)+1$. The decoder $g$ searches the block $Y_{i+1}^{i+M}$ for a synchronization sequence. At most there will be one such sequence. If it detects one string $\mathbf{S}$, which starts at position $i+r, 1 \leq r \leq n+1$, then it lets $\hat{X}_{i}=$ $U_{n-r+1}$, where $\left[\hat{U}_{1}, \hat{U}_{2}, \ldots, \hat{U}_{n}\right] \triangleq \mathrm{g}_{n}\left(Z_{i+r-1}^{i+r-n}, Y_{i+r-1}^{i+r-n}\right)$. If it detects no synchronization sequence, the decoder outputs some fixed arbitrary symbol.

In order to compute the expected average distortion between the source and reconstruction sequences, note that since the original process and its reconstruction are jointly stationary, the average expected distortion between them is equal to $\mathrm{E} \lambda\left(X_{0}, \hat{X}_{0}\right)$

$$
\begin{aligned}
\mathrm{E} \lambda\left(X_{0}, \hat{X}_{0}\right)= & \sum_{i=0}^{n-1} \mathrm{E}\left[\lambda\left(X_{0}, \hat{X}_{0}\right) \mid T^{i} F\right] \mathrm{P}\left(T^{i} F\right) \\
& +\mathrm{E}\left[\lambda\left(X_{0}, \hat{X}_{0}\right) \mid \bigcup_{i=n}^{L_{n}-1} T^{i} F\right] \mathrm{P}\left(\bigcup_{i=n}^{L_{n}-1} T^{i} F\right) \\
& +\mathrm{E}\left[\lambda\left(X_{0}, \hat{X}_{0}\right) \mid G\right] \mathrm{P}(G) .
\end{aligned}
$$

By the stationarity of the source

$$
\mathrm{P}\left(\bigcup_{i=0}^{L_{n}-1} T^{i} F\right)=(n+\lceil n \epsilon\rceil) \mathrm{P}(F)
$$

and $\mathrm{P}\left(\bigcup_{i=n}^{L_{n}-1} T^{i} F\right)=\lceil n \epsilon\rceil \mathrm{P}(F)$. Therefore

$$
\begin{aligned}
\mathrm{P}\left(\bigcup_{i=n}^{L_{n}-1} T^{i} F\right) & =\frac{\lceil n \epsilon\rceil}{n+\lceil n \epsilon\rceil} \mathrm{P}\left(\bigcup_{i=0}^{L_{n}-1} T^{i} F\right) \\
& \leq \frac{\lceil n \epsilon\rceil}{n+\lceil n \epsilon\rceil} \\
& \stackrel{(a)}{\leq} \epsilon
\end{aligned}
$$

where (a) follows from (A1). Moreover, from the RK Theorem, $\mathrm{P}(G)<\epsilon$, which together with (A8) shows that

$$
\mathrm{E} \lambda\left(X_{0}, \hat{X}_{0}\right)<\sum_{i=0}^{n-1} \mathrm{E}\left[\lambda\left(X_{0}, \hat{X}_{0}\right) \mid T^{i} F\right] \mathrm{P}\left(T^{i} F\right)+2 \lambda_{\max } \epsilon .
$$

For bounding the first term in (A9), note that

$$
\begin{aligned}
& \sum_{i=0}^{n-1} \mathrm{E}\left[\lambda\left(X_{0}, \hat{X}_{0}\right) \mid T^{i} F\right] \mathrm{P}\left(T^{i} F\right) \\
& =\sum_{i=0}^{n-1} \mathrm{E}\left[\lambda\left(X_{i}, \hat{X}_{i}\right) \mid F\right] \mathrm{P}(F) \\
& \quad<\mathrm{E}\left[\rho_{n}\left(X^{n}, \hat{X}^{n}\right) \mid F\right]
\end{aligned}
$$

where the last line follows from the fact that $\mathrm{P}(F)<\frac{1}{n}$. On the other hand, by the RK theorem, $\mathrm{E}\left[\rho_{n}\left(X^{n}, \hat{X}^{n}\right) \mid F\right]=$ $\mathrm{E}\left[\rho_{n}\left(X^{n}, \mathrm{~g}_{n}\left(Z^{n}, \mathrm{f}_{n}\left(X^{n}\right)\right)\right)\right]$. Consequently, combining all of the previous results

$$
\begin{aligned}
\mathrm{E}\left[\lambda\left(X_{0}, \hat{X}_{0}\right)\right]< & \mathrm{E}\left[\rho_{n}\left(X^{n}, \mathrm{~g}_{n}\left(Z^{n}, \mathrm{f}_{n}\left(X^{n}\right)\right)\right)\right] \\
& +2 \lambda_{\max } \epsilon,<D+\left(1+2 \lambda_{\max }\right) \epsilon .
\end{aligned}
$$

From the finite SB code approximation theorem ([36, Theorem 3.1]), for any $\sigma>0$, and any infinite SB code $f^{(\infty)}$, there exists $k=k\left(\sigma, f^{(\infty)}\right)$, and finite SB code $f^{(k)}$ of windowlength $2 k+1$, such that the outputs of the codes $f^{(\infty)}$ and $f^{(k)}$ coincide except on a set of probability no larger than $\sigma$. 
This result enables us to truncate $f^{(\infty)}$, and get a finite code $f^{(k)}$ such that $\mathrm{P}\left(f^{(\infty)}(\mathbf{X}) \neq f^{(k)}(\mathbf{X})\right)<\sigma$. Now assume that $\sigma=\epsilon /(2 M+1)$ and $k=k\left(\sigma, f^{(\infty)}\right)$, and define $\left\{Y_{i}\right\}$ and $\left\{\tilde{Y}_{i}\right\}$ as $Y_{i}=f^{(k)}\left(X_{i-k}^{i+k}\right)$ and $\tilde{Y}_{i}=f^{(\infty)}(\mathbf{X})$. Then, from Lemma 3.2 of [34]

$$
\begin{aligned}
& \mathrm{P}\left(g\left(Z_{i-M}^{i+M}, Y_{i-M}^{i+M}\right) \neq g\left(Z_{i-M}^{i+M}, \tilde{Y}_{i-M}^{i+M}\right)\right) \\
& \quad \leq(2 M+1) \mathrm{P}\left(Y_{i} \neq \tilde{Y}_{i}\right) \\
& <(2 M+1) \sigma \\
& =\epsilon .
\end{aligned}
$$

(A12) states that from the truncation of $f^{\infty}$ the expected distortion will not increase by more than $l_{\max } \epsilon$. Therefore, combing this with our previous results, we conclude that

$$
\mathrm{E}\left[\lambda\left(X_{0}, \hat{X}_{0}\right)\right]<D+\left(1+3 \lambda_{\max }\right) \epsilon .
$$

So far we have shown the existence of a SB code which generates a reconstruction sequence within maximum distance of $D+\left(1+3 \lambda_{\max }\right) \epsilon$ to the source. Now we show that the entropy rate of the $\left\{Y_{i}\right\}$ sequence, where $Y_{i}=f^{(k)}\left(X_{i-k}^{i+k}\right)$, is as close to $R$ as desired. In order to do this, we first bound the entropy rate of the $\left\{\tilde{Y}_{i}\right\}$ process, where $\tilde{Y}_{i}=f^{(\infty)}(\mathbf{X})$. Then

$$
\begin{aligned}
\frac{1}{m} H\left(Y^{m}\right) & \leq \frac{1}{m} H\left(Y^{m}, \tilde{Y}^{m}\right) \\
& =\frac{1}{m} H\left(\tilde{Y}^{m}\right)+\frac{1}{m} H\left(Y^{m} \mid \tilde{Y}^{m}\right) \\
& =\frac{1}{m} H\left(\tilde{Y}^{m}\right)+\frac{1}{m} H\left(Y^{m} \oplus \tilde{Y}^{m} \mid \tilde{Y}^{m}\right) \\
& \leq \frac{1}{m} H\left(\tilde{Y}^{m}\right)+\frac{1}{m} H\left(Y^{m} \oplus \tilde{Y}^{m}\right) \\
& \leq \frac{1}{m} H\left(\tilde{Y}^{m}\right)+h_{b}(\sigma)
\end{aligned}
$$

where for $y, \tilde{y} \in \mathcal{Y}, y \oplus \tilde{y}=0$ if $y=\tilde{y}$ and 1 , otherwise, also for $0 \leq \alpha \leq 1, h_{b}(\alpha)=-\alpha \log \alpha-(1-\alpha) \log (1-\alpha)$. Therefore, letting $m$ grow to infinity, we conclude that

$$
H(\mathbf{Y}) \leq H(\tilde{\mathbf{Y}})+h_{b}(\sigma) .
$$

Now we turn to bounding $H(\tilde{\mathbf{Y}})$. Let $\left\{\theta_{i}\right\}$ denote a sequence defined as follows:

$$
\theta_{i}= \begin{cases}j, & \tilde{Y}_{i} \text { is the } j^{\text {th }} \text { letter of a codeword } \\ & \text { where } j \in\left\{1, \ldots, L_{n}\right\} \\ 0, & \text { otherwise }\end{cases}
$$

Then the entropy rate of the generated FB process $\tilde{\mathbf{Y}}$ can be upper-bounded as follows:

$$
\begin{aligned}
H(\tilde{\mathbf{Y}}) & =\lim _{m \rightarrow \infty} \frac{1}{m} H\left(\tilde{Y}^{m}\right) \\
& \leq \lim _{m \rightarrow \infty} \frac{1}{m} H\left(\tilde{Y}^{m}, \theta^{m}\right) \\
& =\lim _{m \rightarrow \infty} \frac{1}{m}\left[H\left(\theta^{m}\right)+H\left(\tilde{Y}^{m} \mid \theta^{m}\right)\right] \\
& =\lim _{m \rightarrow \infty}\left[\frac{1}{m} H\left(\theta^{m}\right)+H\left(\tilde{Y}_{m} \mid \tilde{Y}^{m-1}, \theta^{m}\right)\right] \\
& \leq \lim _{m \rightarrow \infty}\left[\frac{1}{m} H\left(\theta^{m}\right)+H\left(\tilde{Y}_{m} \mid \tilde{Y}^{m-1}, \theta_{m}\right)\right]
\end{aligned}
$$

where

$$
\begin{aligned}
& H\left(\tilde{Y}_{m} \mid \tilde{Y}^{m-1}, \theta_{m}\right) \\
& =\sum_{j=0}^{L_{n}} H\left(\tilde{Y}_{m} \mid \tilde{Y}^{m-1}, \theta_{m}=j\right) \mathrm{P}\left(\theta_{m}=j\right) \\
& \stackrel{(a)}{\leq} \epsilon \log |\mathcal{Y}|+\sum_{j=1}^{n} H\left(\tilde{Y}_{m} \mid \tilde{Y}_{m-j+1}^{m-1}, \theta_{m}=j\right) \mathrm{P}\left(\theta_{m}=j\right) \\
& \leq \epsilon \log |\mathcal{Y}|+\frac{1}{n} \sum_{j=1}^{n} H\left(\tilde{Y}_{m} \mid \tilde{Y}_{m-j+1}^{m-1}, \theta_{m}=j\right) \\
& =\epsilon \log |\mathcal{Y}|+\frac{1}{n} H\left(\mathrm{f}_{n}\left(X^{n}\right)\right) \\
& \leq \epsilon \log |\mathcal{Y}|+R_{1}
\end{aligned}
$$

where (a) follows from the facts that, for $n+1 \leq j \leq L_{n}$, $H\left(\tilde{Y}_{m} \mid \tilde{Y}^{m-1}, \theta_{m}=j\right)=0$, and $\mathrm{P}\left(\theta_{m}=0\right)<\epsilon$. Moreover, we show that the entropy rate of the $\left\{\theta_{i}\right\}$ process can be made arbitrary small:

$$
\begin{aligned}
\lim _{m \rightarrow \infty} \frac{1}{m} H\left(\theta^{m}\right) & =\lim _{m \rightarrow \infty} H\left(\theta_{m} \mid \theta^{m-1}\right) \\
& \leq \lim _{m \rightarrow \infty} H\left(\theta_{m} \mid \theta_{m-1}\right) \\
& =\sum_{j=0}^{L_{n}} \mathrm{P}\left(\theta_{0}=j\right) H\left(\theta_{1} \mid \theta_{0}=j\right)
\end{aligned}
$$

where the last step is a result of stationarity. By the definition of the $\left\{\theta_{i}\right\}$ sequence, $H\left(\theta_{1} \mid \theta_{0}=j\right)=0$, for $1 \leq j \leq n-1$, $\mathrm{P}\left(\theta_{0}=0\right)=\mathrm{P}(G) \leq \epsilon$, and $\mathrm{P}\left(\theta_{0}=L_{n}\right)=\mathrm{P}(F) \leq \frac{1}{n}$. Given $\theta_{0}=0, \theta_{1}$ can either be zero or one, therefore $H\left(\theta_{1} \mid \theta_{0}=0\right) \leq$ 1. Similarly, conditioned on $\theta_{0}=L_{n}, \theta_{1}$ can only be zero or one, and $H\left(\theta_{1} \mid \theta_{0}=n\right)$ computes the uncertainty that one has in determining whether a sequence belongs to $\bigcup_{i=0}^{L_{n}} T^{i} F$ or not when it is known that $\mathbf{X} \in T^{-L_{n}} F$. Since conditioning can only reduce entropy, and $\mathrm{P}(G)<\epsilon$, it follows that $H\left(\theta_{1} \mid \theta_{0}=\right.$ $\left.L_{n}\right)<h_{b}(\epsilon)$. Consequently

$$
\begin{aligned}
\lim _{m \rightarrow \infty} \frac{1}{m} H\left(\theta^{m}\right)= & \mathrm{P}\left(\theta_{0}=0\right) H\left(\theta_{1} \mid \theta_{0}=0\right) \\
& +\mathrm{P}\left(\theta_{0}=L_{n}\right) H\left(\theta_{1} \mid \theta_{0}=L_{n}\right) \\
\leq & \epsilon+\frac{1}{n} h_{b}(\epsilon) .
\end{aligned}
$$

Combining (A15), (A17), and (A20), it follows that

$$
H(\mathbf{Y}) \leq R_{1}+(1+\log |\mathcal{Y}|) \epsilon+\frac{1}{n} h_{b}(\epsilon)+h_{b}(\sigma)
$$

where as defined before

$$
\sigma=\frac{\epsilon}{2(n+\lceil n \epsilon\rceil)+1} .
$$

Note that $(1+\log |\mathcal{Y}|) \epsilon+\frac{1}{n} h_{b}(\epsilon)+h_{b}(\sigma)$ goes to zero as $\epsilon$ goes to zero. Therefore, there exists $\epsilon^{\prime}>0$, such that $\left(\frac{1}{n}+\right.$ $\log |\mathcal{Y}|) \epsilon+\frac{1}{n} h_{b}(\epsilon)+h_{b}(\sigma)<\frac{\delta}{2}$, for any $\epsilon<\epsilon^{\prime}$. By definition $R_{1}=R-\delta$. Consequently, by choosing $\epsilon<\min \left\{\epsilon^{\prime}, \epsilon^{\prime \prime}\right\}$, where $\epsilon^{\prime \prime} \triangleq \frac{\epsilon_{1}}{1+3 \lambda_{\max }}$, and $\epsilon_{2} \triangleq \frac{\delta}{2}>0$, we get a SB encoder $f^{(k)}$ and SB decoder $g$ generating FB and reconstruction sequences satisfying 
1) $\mathrm{E}\left[\lambda\left(X_{0}, \hat{X}_{0}\right)\right]<D+\epsilon_{1}$,

2) $H(\mathbf{Y})<R-\epsilon_{2}$.

\section{APPENDIX B}

PROOF OF THEOREM 5.1

First, we prove that for any given $\epsilon>0$, there exists $N_{\epsilon}>0$, such that for $n>N_{\epsilon}$

$$
\mathrm{E}\left[\rho_{n}\left(X^{n}, \mathrm{~g}_{n}^{*}\left(Z^{n}, \mathrm{f}_{n}^{*}\left(X^{n}\right)\right)\right)\right]<D_{\mathbf{X}, \boldsymbol{\pi}}(R)+\epsilon .
$$

By definition, $D_{\mathbf{X}, \pi}(R)$ denotes the infimum of all distortions achievable by WZ coding of source $\mathbf{X}$ at rate $R$ when the DMC is described by $\pi$. Therefore, for any $\epsilon>0,\left(D+\frac{\epsilon}{4}, R\right)$ would be an interior point of the rate-distortion region. Hence, by theorem 4.1 for $\epsilon_{1}=\frac{\epsilon}{4}>0$, there exist some $\epsilon_{2}>0$, and a slidingblock WZ code with mappings $f$ and $g$, each one having a finite window length, such that

1) $\mathrm{E}\left[\lambda\left(X_{i}, g\left(Z_{i-l}^{i+l}, Y_{i-m}^{i+m}\right)\right)\right] \leq D+\frac{\epsilon}{2}$, where $Y_{i}=$ $f\left(X_{i-k}^{i+k}\right)$,

2) $H(\mathbf{Y})=\lim _{n \rightarrow \infty} \frac{1}{n} H\left(Y_{1}, \ldots, Y_{n}\right) \leq R-\epsilon_{2}$, for some $\epsilon_{2}>0$.

On the other hand, the FB process $\left\{Y_{i}\right\}$ generated by sliding windowing a stationary ergodic process $\left\{X_{i}\right\}$ with a time invariant mapping $f$, is also a stationary ergodic process. Consequently, since for any stationary ergodic process Lempel-Ziv coding algorithm is an asymptotically optimal lossless compression scheme [37], for any given $\sigma>0$, there exists $N_{\sigma}>0$, such that for $n>N_{\sigma}$,

$$
\frac{1}{n} \mathrm{LZ}\left(Y_{1}, \ldots, Y_{n}\right) \leq H(\mathbf{Y})+\sigma .
$$

Letting $\sigma=\frac{\epsilon_{2}}{2}$, and choosing $n$ greater than the corresponding $N_{\sigma}$, yields

$$
\frac{1}{n} \mathrm{LZ}\left(Y_{1}, \ldots, Y_{n}\right) \leq R-\epsilon_{2}+\sigma, \leq R-\frac{\epsilon_{2}}{2},<R .
$$

Therefore, for any given $\epsilon>0$, and any source output sequence, by choosing the block length $n$ sufficiently large, the mapping $f$ would belong to $S\left(x^{n}, k_{n}, R\right)$. On the other hand, since for any individual source sequence $x^{n}, f^{*}$ is the mapping in $S$ that defines the FB sequence minimizing the expected distortion, it follows that

$$
V\left(f^{*}, l, m\right)<V(f, l, m) .
$$

Moreover, since $V\left(f^{*}, l, m\right)$ is the minimum accumulated loss attainable by the mappings in $S(n, l, m)$, when the decoder is constrained to be a sliding window decoder with parameters $l$ and $m$, it is in turns less than the expected distortion obtained by the specific mapping $g$ given by Theorem 4.1, i.e.

$$
\begin{aligned}
& \mathrm{E}\left[\sum_{i=k+1}^{n-k} \lambda\left(x_{i}, g^{*}\left(Z_{i-l}^{i+l}, \tilde{y}_{i-m}^{i+m}\right)\right)\right] \\
& \leq \mathrm{E}\left[\sum_{i=k+1}^{n-k} \lambda\left(x_{i}, g\left(Z_{i-l}^{i+l}, y_{i-m}^{i+m}\right)\right)\right]
\end{aligned}
$$

where $y_{i}=f\left(x_{i-k}^{i+k}\right)$ and $\tilde{y}_{i}=f^{*}\left(x_{i-k}^{i+k}\right)$.

The final step is applying Theorem 3.1 which is the asymptotic optimality of WZ DUDE algorithm in the semistochastic setting. From this result, when the parameter $l$ and $m$ are such that $t_{n}=\max \{l, m\}=o(n / \log n)$, the difference between the performance of the WZ DUDE decoding algorithm and the optimal sliding-window decoder of the same order goes to zero as the block length goes to infinity. In other words, for any given $\epsilon>0$, there exists $N_{\epsilon}^{\prime}>0$, such that for $n>N_{\epsilon}^{\prime}$

$$
\begin{aligned}
& \frac{1}{n-2 k} \mathrm{E}\left[\sum_{i=k+1}^{n-k} \lambda\left(x_{i}, \hat{x}_{i}\right)\right] \\
& \leq \frac{1}{n-2 k} \mathrm{E}\left[\sum_{i=k+1}^{n-k} \lambda\left(x_{i}, g^{*}\left(Z_{i-l}^{i+l}, \tilde{y}_{i-m}^{i+m}\right)\right)\right]+\frac{\epsilon}{4}
\end{aligned}
$$

where $\hat{x}^{n}=\mathrm{g}_{n}^{*}\left(Z^{n}, \mathrm{f}_{n}^{*}\left(X^{n}\right)\right)$. Note that the only uncertainty in (B5) is due to the channel noise, and the source and FB sequence are assumed to be individual sequences. Combining (B4) and (B5), it follows that with probability one

$$
\begin{aligned}
& \frac{n}{n-2 k} \mathrm{E}\left[\rho_{n}\left(x^{n}, \mathrm{~g}_{n}^{*}\left(Z^{n}, \mathrm{f}_{n}^{*}\left(X^{n}\right)\right)\right)\right] \\
& \quad \leq \frac{1}{n-2 k} \mathrm{E}\left[\sum_{i=k+1}^{n-k} \lambda\left(x_{i}, g\left(Z_{i-l}^{i+l}, y_{i-m}^{i+m}\right)\right)\right]+\frac{\epsilon}{4} .
\end{aligned}
$$

On the other hand, since $\left\{\left(X_{i}, Y_{i}\right)\right\}_{-\infty}^{\infty}$ is also a stationary ergodic process with super-alphabet $\mathcal{X} \times \mathcal{Y}$, by the ergodic theory, with probability one

$$
\begin{aligned}
& \lim _{n \rightarrow \infty} \frac{1}{n-2 k} \sum_{i=k+1}^{n-k} \mathrm{E}\left[\lambda\left(x_{i}, g\left(Z_{i-l}^{i+l}, y_{i-m}^{i+m}\right)\right)\right] \\
& =\mathrm{E}\left[\lambda\left(X_{0}, g\left(Z_{-l}^{l}, Y_{-m}^{m}\right),\right)\right] \\
& \leq D_{\mathbf{X}, \boldsymbol{\pi}}+\frac{\epsilon}{2} .
\end{aligned}
$$

This means that with probability one, there exists $N_{\epsilon}^{\prime \prime}>0$, such that for $n>N_{\epsilon}^{\prime \prime}$,

$$
\begin{aligned}
\frac{1}{n-2 k} \sum_{i=k+1}^{n-k} \mathrm{E}\left[\lambda\left(x_{i}, g\left(Z_{i-l}^{i+l}, y_{i-m}^{i+m}\right)\right)\right] & \\
& \leq D_{\mathbf{X}, \boldsymbol{\pi}}+\frac{\epsilon}{2}+\frac{\epsilon}{4} .
\end{aligned}
$$

Finally, combining (B6) and (B7), and taking $n>N_{\epsilon}$, where $N_{\epsilon}=\max \left\{N_{\sigma}, N_{\epsilon}^{\prime}, N_{\epsilon}^{\prime \prime}\right\}$, yields the desired result as follows:

$$
\frac{n}{n-2 k} \mathrm{E}\left[\rho_{n}\left(X^{n}, \mathrm{~g}_{n}^{*}\left(Z^{n}, \mathrm{f}_{n}^{*}\left(X^{n}\right)\right)\right)\right], \leq D_{\mathbf{X}, \boldsymbol{\pi}}(R)+\epsilon \text {. }
$$

\section{ACKNOWLEDGMENT}

The authors would like to thank E. Ordentlich and P. Vontobel for the helpful discussions. The authors also would like to thank the anonymous reviewers for their helpful comments.

\section{REFERENCES}

[1] A. Wyner and J. Ziv, "The rate-distortion function for source coding with side information at the decoder," IEEE Trans. Inf. Theory, vol. 1 , pp. 1-10, Jan. 1976.

[2] S. Jalali and T. Weissman, "Lossy coding via Markov chain Monte Carlo," in Proc. 2008 IEEE Int. Symp. Inf. Theory, Toronto, ON Canada, Jul. 2008.

[3] S. Jalali, A. Montanari, and T. Weissman, "An implementable scheme for universal lossy compression of discrete Markov sources," in Proc. 2009 Data Compression Conf., Snowbird, UT, Mar. 2009.

[4] A. Gupta, S. Verdú, and T. Weissman, "Rate-distortion in near-linear time," IEEE Trans. Inf. Theory. 
[5] R. M. Gray, "Rate distortion functions for finite-state finite-alphabet Markov sources," IEEE Trans. Inf. Theory, vol. 17, no. 2, pp. 127-134, Mar. 1971.

[6] T. Berger, "Explicit bounds to R(D) for a binary symmetric Markov source," IEEE Trans. Inf. Theory, vol. 23, no. 1, pp. 52-59, Jan. 1977.

[7] S. Jalali and T. Weissman, "New bounds on the rate-distortion function of a binary Markov source," in Proc. 2007 IEEE Int. Symp. Inf. Theory, Nice, France, Jul. 2007.

[8] S. Shamai, S. Verdú, and R. Zamir, "The rate-distortion function for source coding with side information at the decoder," IEEE Trans. Inf. Theory, vol. 44, no. 2, pp. 564-579, Mar. 1998.

[9] S. Venbu, S. Verdú, and Y. Steinberg, "The source-channel separation theorem revisited," IEEE Trans. Inf. Theory, vol. 41, no. 1, pp. 44-54, Jan. 1995.

[10] J. Ziv and A. Lempel, "Compression of individual sequences via variable-rate coding," IEEE Trans. Inf. Theory, vol. 24, no. 5, pp. 530-536, Sep. 1978.

[11] R. M. Gray, D. L. Neuhoff, and D. S. Ornstein, "Nonblock source coding with a fidelity criterion," The Ann. Probab., vol. 3, no. 3, pp. 478-491, Jun. 1975.

[12] T. Weissman, E. Ordentlich, G. Seroussi, S. Verdú, and M. Weinberger, "Universal discrete denoising: Known channel," IEEE Trans. Inf. Theory, vol. 51, no. 1, pp. 5-28, Jan. 2005.

[13] G. M. Gemelos, S. Sigurjonsson, and T. Weissman, "Universal minimax discrete denoising under channel uncertainty," IEEE Trans. Inf. Theory, vol. 52, no. 8, pp. 3476-3497, Aug. 2006.

[14] G. M. Gemelos, S. Sigurjonsson, and T. Weissman, "Algorithms for discrete denoising under channel uncertainty," IEEE Trans. Signal Process., vol. 54, no. 6, pp. 2263-2276, Jun. 2006.

[15] B. Girod, A. Aaron, S. Rane, and D. Rebollo-Monedero, "Distributed video coding," IEEE Trans. Inf. Theory, vol. 93, no. 1, pp. 71-83, Jan. 2005.

[16] S. S. Pradhan and K. Ramchandran, "Distributed source coding using syndromes (DISCUS)," IEEE Trans. Inf. Theory, vol. 49, no. 3, pp. 626-643, Mar. 2003.

[17] D. Rebollo-Monedero, R. Zhang, and B. Girod, "Design of optimal quantizers for distributed source coding," in Proc. 2000 Data Compress. Conf., Snowbird, UT, Mar. 2003.

[18] S. D. Servetto, "Lattice quantization with side information," in Proc. 2000 Data Compress. Conf., Snowbird, UT, Mar. 2000.

[19] Y. Yang, S. Cheng, Z. Xiong, and W. Zhao, "Wyner-Ziv coding based on TCQ and LDPC codes," in Proc. 2003 Asilomar Conf. Signals, Syst., Comput., Pacific Grove, CA, Nov. 2003.

[20] R. Z. S. Shamai and U. Erez, "Nested linear/lattice codes for structured multiterminal binning," IEEE Trans. Inf. Theory, vol. 48, no. 6, pp. 1250-1276, Jun. 2002.

[21] N. Merhav and J. Ziv, "On the Wyner-Ziv problem for individual sequences," IEEE Trans. Inf. Theory, vol. 52, no. 3, pp. 867-873, Mar. 2006.

[22] J. Yu and S. Verdú, "Schemes for bidirectional modeling of discrete stationary sources," IEEE Trans. Inf. Theory, vol. 52, no. 11, pp. 4789-4807, Nov. 2006.

[23] E. Ordentlich, M. J. Weinberger, and T. Weissman, "Multi-directional context sets with applications to universal denoising and compression," in Proc. 2005 IEEE Int. Symp. Inf. Theory, Adelaide, Australia, Sep. 2005, pp. 1270-1274.

[24] E. Ordentlich, M. J. Weinberger, and T. Weissman, "Efficient pruning of bi-directional context trees with applications to universal denoising and compression," in Proc. 2004 IEEE Int. Symp. Inf. Theory, San Antonio, TX, Oct. 2004.

[25] K. Marton, "On the rate distortion function of stationary sources," Probl. Contr. Inf. Theory, vol. 4, pp. 289-297, 1975.

[26] J. Kieffer, "A method for proving multiterminal source coding theorems," IEEE Trans. Inf. Theory, vol. 27, no. 5, pp. 565-570, 1981.

[27] T. Berger, Multiterminal Source Coding. New York: SpringerVerlag, 1977.

[28] A. Kaspi and T. Berger, "Rate-distortion for correlated sources with partially separated encoders," IEEE Trans. Inf. Theory, vol. 28, no. 6, pp. 828-840, Nov. 1982.

[29] S. Y. Tung, "Multiterminal source coding," Ph.D., Cornell Univ., Ithaca, NY, 1977.

[30] E.-H. Yang and J. C. Kieffer, "Simple universal lossy data compression schemes derived from the Lempel-Ziv algorithm," IEEE Trans. Inf. Theory, vol. 42, no. 1, pp. 239-245, Jan. 1996.
[31] K. Sivaramakrishnan and T. Weissman, "Universal denoising of discrete-time continuous-amplitude signals," IEEE Trans. Inf. Theory, vol. 54, no. 12, pp. 5632-5660, Dec. 2008.

[32] K. Sivaramakrishnan and T. Weissman, "A context quantization approach to universal denoising," IEEE Trans. Signal Process., vol. 57, no. 6, pp. 2110-2129, Jun. 2009.

[33] T. Moon and T. Weissman, "Discrete denoising with shifts," IEEE Trans. Inf. Theory, Aug. 2007.

[34] R. M. Gray, Block, Sliding-Block, and Trellis Codes. Keszthely, Hungary: Janos Bolyai Colloquiem on Inf. Theory, 1975.

[35] P. C. Shields, The Theory of Bernoulli Shifts. Chicago, IL: Univ. Chicago Press, 1973.

[36] R. Gray, "Sliding-block source coding," IEEE Trans. Inf. Theory, vol. 21, no. 4, pp. 357-368, Jul. 1975.

[37] T. M. Cover and J. A. Thomas, Elements of Information Theory, 2nd ed. New York: Wiley, 2006

Shirin Jalali (S'05) received the B.S. and M.S. degrees, both in electrical engineering, from Sharif University of Technology, Tehran, Iran, in 2002 and 2004, respectively. She received the M.S. degree in statistics and the Ph.D. degree in electrical engineering from Stanford University, Stanford, CA, in 2009.

Her research interests are information theory and statistical signal processing. She has recently joined the Center for Mathematics of Information, California Institute of Technology, Pasadena, as a Postdoctoral Fellow.

Sergio Verdú (S'80-M'84-SM'88-F'93) received the Telecommunications Engineering degree from the Universitat Politècnica de Barcelona, Spain, in 1980 and the Ph.D. degree in electrical engineering from the University of Illinois at Urbana-Champaign in 1984.

Since 1984, he has been a member of the faculty of Princeton University, Princeton, NJ, where he is the Eugene Higgins Professor of Electrical Engineering. He is the author of "Multiuser Detection" (Cambridge, U.K.: Cambridge University Press, 1998), which received the 2000 Frederick E. Terman Award from the American Society for Engineering Education.

Dr. Verdú is the recipient of the 2007 Claude E. Shannon Award and the 2008 IEEE Richard W. Hamming Medal. He is a member of the National Academy of Engineering and was awarded a Doctorate Honoris Causa from the Universitat Politècnica de Catalunya in 2005. He has received several paper awards from the IEEE: the 1992 Donald Fink Paper Award, the 1998 Information Theory Outstanding Paper Award, an Information Theory Golden Jubilee Paper Award, the 2002 Leonard Abraham Prize Award, the 2006 Joint Communications/Information Theory Paper Award, and the 2009 Stephen O. Rice Prize from IEEE Communications Society. He has also received paper awards from the Japanese Telecommunications Advancement Foundation and from EURASIP. He served as President of the IEEE Information Theory Society in 1997. He is currently Editor-in-Chief of Foundations and Trends in Communications and Information Theory.

Tsachy Weissman (SM'03) received the undergraduate and graduate degrees from the Department of Electrical Engineering, the Technion-Israel Institute of Technology, Haifa.

Following his graduation, he has held a faculty position at the Technion, and Postdoctoral appointments with the Statistics Department, Stanford University, Stanford, CA, and with Hewlett-Packard Laboratories. Since summer 2003, he has been on the faculty of the Department of Electrical Engineering, Stanford University, spending the academic years 2007-2009 on leave with the Department of Electrical Engineering, Technion. His research interests span information theory and its applications, and statistical signal processing. He is inventor or co-inventor of several patents in these areas and involved in a number of high-tech companies as a researcher or member of the technical board.

Dr. Weissman has received the NSF CAREER Award, a Horev fellowship for leaders in Science and Technology, and the Henry Taub prize for excellence in research. He is a Robert N. Noyce Faculty Scholar of the School of Engineering at Stanford, and a recipient of the 2006 IEEE joint IT/COM Societies Best Paper Award. 